\title{
Advances in Hybrid Polymer-Based Materials for Sustained Drug Release
}

\author{
Lígia N. M. Ribeiro, ${ }^{1}$ Ana C. S. Alcântara, ${ }^{2}$ Gustavo H. Rodrigues da Silva, ${ }^{1}$ \\ Michelle Franz-Montan, ${ }^{3}$ Silvia V. G. Nista, ${ }^{4}$ Simone R. Castro, ${ }^{1}$ Verônica M. Couto, ${ }^{1}$ \\ Viviane A. Guilherme, ${ }^{1}$ and Eneida de Paula ${ }^{1}$ \\ ${ }^{1}$ Department of Biochemistry and Tissue Biology, Biology Institute, University of Campinas, 13083-862 Campinas, SP, Brazil \\ ${ }^{2}$ Department of Chemistry, Federal University of Maranhão, 65080-805 São Luís, MA, Brazil \\ ${ }^{3}$ Department of Physiological Sciences, Piracicaba Dental School, University of Campinas, 13414-903 Piracicaba, SP, Brazil \\ ${ }^{4}$ Department of Materials and Bioprocess Engineering, School of Chemical Engineering, University of Campinas, \\ 13083-852 Campinas, SP, Brazil
}

Correspondence should be addressed to Lígia N. M. Ribeiro; nuneslica@gmail.com and Eneida de Paula; depaula@unicamp.br

Received 7 August 2017; Accepted 4 October 2017; Published 1 November 2017

Academic Editor: Chaoqun Zhang

Copyright ( 2017 Lígia N. M. Ribeiro et al. This is an open access article distributed under the Creative Commons Attribution License, which permits unrestricted use, distribution, and reproduction in any medium, provided the original work is properly cited.

\begin{abstract}
The use of biomaterials composed of organic pristine components has been successfully described in several purposes, such as tissue engineering and drug delivery. Drug delivery systems (DDS) have shown several advantages over traditional drug therapy, such as greater therapeutic efficacy, prolonged delivery profile, and reduced drug toxicity, as evidenced by in vitro and in vivo studies as well as clinical trials. Despite that, there is no perfect delivery carrier, and issues such as undesirable viscosity and physicochemical stability or inability to efficiently encapsulate hydrophilic/hydrophobic molecules still persist, limiting DDS applications. To overcome that, biohybrid systems, originating from the synergistic assembly of polymers and other organic materials such as proteins and lipids, have recently been described, yielding molecularly planned biohybrid systems that are able to optimize structures to easily interact with the targets. This work revised the biohybrid DDS clarifying their advantages, limitations, and future perspectives in an attempt to contribute to further research of innovative and safe biohybrid polymer-based system as biomaterials for the sustained release of active molecules.
\end{abstract}

\section{Introduction}

This work demonstrates the wide possibility of advanced materials developments in the drug delivery/release field based on a molecular approach. It draws a parallel line between the nanostructured drug delivery systems (DDS) or drug release systems studied in recent years and their hybridization processes from an assembly with polymeric materials able to bypass many of the intrinsic limitations to each system. These versatile polymer-based hybrid nanostructures combine the advantages of each excipient, resulting in formulations or pharmaceutical forms designed to specifically interact with the targets, acting as smart drug delivery systems. The current literature revision aims to show the mainly polymeric materials used as DDS, as well as those organic hybrid nanocarriers based on polymer molecules. We have also moved efforts to inspire novel hybrid DDS in the search of a perfect DDS design, which is still a challenge.

The pioneering use of biomaterials dates back to early civilizations, such as Ancient Egypt, since artificial ears, noses, and teeth were found in many mummified bodies [1]. In the last 50 years, much effort has been focused on understanding the interactions between biomaterials and targets, contributing to the creation of advanced products with different biomedical applications [1]. Polymeric blends as well as the combination of different types of biomaterials 
have been tried for the development of biosensors, scaffolds, implants, tissue engineering, wound healing, and sustained drug delivery [2].

The so-called DDS are technological devices that have been studied since 1959 to overcome the limitations of the traditional drug therapy [3]. DDS are composed of excipients such as inorganic, lipid, or polymeric origin (or their combination), used in the preparation of nanosystems (e.g., vesicles, micelles, nanocapsules, and nanospheres), microparticulates (e.g., microspheres and microemulsions), or dosage forms, such as films and hydrogels. Each carrier system has specific properties and can bind, encapsulate, adhere to, or adsorb active compounds such as genes or drugs [4]. Among the desirable features of DDS are nontoxicity, high drug upload, and targeting, thus reducing side effects in nontarget tissues, sustained delivery of therapeutic molecules, physicochemical stability, ability to protect drugs from degradation, sterilization, and large-scale production [3-5].

One of the first lipid-based DDS described in the literature were liposomes, in 1963, being to this day one of the most studied nanocarriers. The evolution and technological improvement led almost 30 years later to the development of solid lipid nanoparticles (SLN, in 1990) and nanostructured lipid carriers (NLC, in 2000), with greater stability and ability to efficiently encapsulate hydrophobic drugs than liposomes [6].

Among polymeric carriers, cyclodextrins (CD) have unique inclusion complex formation properties, even though their use as carriers (1997) was only described 100 years after their isolation [7]. In addition to CD, other polymeric carriers were later described, capable of forming micro- or nanoparticles (nanocapsules and nanospheres) that require the use of high amount of organic solvents in the preparation method [8]. However, many of these above-mentioned DDS systems may not provide specific properties such as adhesion, fixation, proper consistency, and viscosity; these properties are required for several administration routes, where preservation of the dosage form at the target site, permeation, and suitable mechanical properties are essential for the efficient delivery of active molecules. In this context, the use of nanocarriers systems combined with (bio)polymeric materials may overcome such limitations.

Thus, organic-organic biohybrid systems for sustained drug release emerged as a versatile strategy for the development of optimized formulations and dosage forms. Biohybrid materials are advanced systems that combine the properties of organic nanocarriers with the adhesiveness and biodegradability of (bio)polymers [9]. Within this context, this minireview aims to examine the main advances in biohybrid materials based on organic compounds for sustained drug release and provides a perspective to inspire their future developments.

\section{Polymeric Matrices for Sustained Release of Active Molecules}

When choosing the matrix in the development of a pharmaceutical form, properties such as versatility, biocompatibility, biodegradability, high drug retention capacity, and low cost should be considered. In this sense, for over 50 years, several types of polymeric materials have been used in biomedical applications [10]. The flexibility to design and select polymers with desirable characteristics ensures their use in a wide variety of applications. Compared to other types of biomaterials, polymers offer wide structural diversity and distinct properties [11]. Polymers used as matrices for sustained drug delivery may be synthetic, natural, or a combination of both. Synthetic polymers include aliphatic polyesters of hydroxy acids, such as polylactic acid (PLA), polylactic-co-glycolic acid (PLGA), or polyvinyl acid (PVA). Among polymers of natural origin (biopolymers) are polypeptides, proteins (e.g., albumin, zein, fibrinogen, gelatin, and collagen), and polysaccharides (e.g., hyaluronic acid, alginate, pectin, and chitosan) [12-14].

In any case, and regardless of origin and chemical nature, the essential feature for a polymer to be used as a matrix in sustained drug release is its biocompatibility. This means that the polymer should not stimulate or cause any kind of allergic reaction or inflammatory response when in contact with living tissues or organic fluids [15]. In this sense, biodegradable polymeric matrices, known as biopolymers, are one of the most promising alternatives in the design of new sustained DDS. As they are biocompatible and biodegradable, the body through usual metabolic processes can excrete them. In addition, they are extremely versatile and can be processed in the most varied forms, such as films, beads, and foams $[16,17]$.

Among the most commonly used biopolymers in DDS, polysaccharides stand out for their abundance, structural diversity, versatility, desirable physicochemical properties, easy handling, and low cost. From the chemical point of view, polysaccharides combine large and distinct carbohydrates, which may be composed of a single kind of monosaccharide or two or more different monomeric units. The ability of polysaccharides to respond to different stimuli such as $\mathrm{pH}$, temperature, ionic strength, and concentration makes them particularly attractive for the development of innovative delivery matrices. Additionally, polysaccharides can be functionalized, which gives them an overall positive or negative charge. The presence of such positively or negatively charged groups is extremely useful in preparing networks of stable gels through cross-linking reactions. Thus, polysaccharide networks can be obtained by cross-linking assisted by interactions of diverse forces (from weak physical forces to irreversible covalent bonds) [18].

In this perspective, chitosan, the second most abundant polysaccharide on the planet (after cellulose), is the only natural polysaccharide positively charged, obtained from the partial alkaline deacetylation of chitin. The level of chitosan deacetylation is a critical parameter in its ability to respond to stimuli. Chitosan-free amine groups are responsible for its sensitiveness to $\mathrm{pH}$ and for high affinity to mammalian cell components and bactericidal activity [15]. Such amine groups may interact with opposite charges of drugs, polymers, or cross-linking agents, such as glutaraldehyde, formaldehyde, genipin, or oxalic acid, used to prepare covalently cross-linked networks, or to either calcium phosphate, $\beta$-glycerophosphate, citrate, or tripolyphosphate, which are employed to generate ionically cross-linked 
hydrogels [19-21]. Thus, studies simulating the gastrointestinal tract showed that chitosan networks present a typical swelling, which is related to the $\mathrm{pH}$ of the medium: high sensitivity in acid medium (high degree of swelling) and low sensitivity in neutral and alkaline medium (low degree of swelling). Such behavior is strategic in the development of dosage forms in which the drug of interest must be absorbed in acidic $\mathrm{pH}$ milieu, being quickly dispersed in the medium. This is the case, for example, of chitosan systems processed as films and cross-linked with different multivalent phosphates for the sustained delivery of riboflavin [22] or of chitosan microspheres cross-linked with glutaraldehyde or epichlorohydrin for release of sodium diclofenac [23]. Some studies have been reported for microspheres prepared from chitosan derivatives, such as malonylchitosan crosslinked with glutaraldehyde for the delivery of acyclovir in the treatment of infections caused by Herpes zoster virus strains [24].

However, in order to increase the resistance of chitosanbased systems to acidic $\mathrm{pH}$, allowing the sustained drug delivery of interest into the digestive system, a commonly employed strategy is the combination of chitosan with other biopolymers that act as macromolecular cross-linking agents. Among them, the polysaccharide alginate was one of the first used in chitosan-based dosage blends. The alginatechitosan blend is widely used as a drug carrier in the most varied designs and purposes [16]. In this sense, nitrofurantoin encapsulated in chitosan-calcium alginate microcapsules proved to have a better-sustained release profile than the antibiotic in pure chitosan particles [25]. The success of the association between these two polysaccharides is the result of a highly synergistic effect of polyelectrolyte-type interactions generated between the protonated amine groups of chitosan and the carboxyl groups of alginate. The combination of chitosan and alginate served as the inspiration for the development of new blends employing other biopolymers, such as glucomannan [26] or silk fibroin [27]. In the latter case, the carrier properties of the chitosan-silk fibroin systems as carriers of four model drugs (theophylline, sodium diclofenac, amoxicillin, and salicylic acid) were evaluated. In vitro delivery studies, performed at $37^{\circ} \mathrm{C}$ in buffer solutions at $\mathrm{pH} 2.0,5.5$, and 7.2, revealed that systems processed as films containing $80 \%$ chitosan delivered at $\mathrm{pH} 2.0$ the maximum amount of all model drugs studied ( $90 \%)$. For the $\mathrm{pH}$ that simulated the intestinal region (7.2), a sustained release profile was evidenced due to the amount of silk fibroin in the system's composition [27].

The combination of chitosan with synthetic polymeric matrices can be employed to increase the biocompatibility of these systems. Thus, drug delivery devices for gingival treatments were developed from the combination of chitosan with polyvinyl acid (PVA), polyethylene glycol (PEG), and polyvinylpyrrolidone (PVP) [28]. FTIR analyses and stress tests in materials processed as films indicated that chitosan-PEG and chitosan-PVP blends presented miscibility in all the studied proportions, while the chitosan-PVA blend only showed interaction at 50:50 and 80:20 ratios (chitosan:PVA). This study also suggested that chitosan blends possessed improved properties when compared to pure chitosan, being a promising alternative for oral mucosal DDS.

Another polysaccharide widely used as matrix in drug delivery systems is alginate, extracted from brown marine algae [29]. Unlike chitosan, this biomacromolecule has carboxylic functional groups, resulting in an overall negative charge. In this case, the usual method of forming alginate networks is to cross-link the gel with bivalent cations, such as $\mathrm{Ca}^{2+}$, forming a three-dimensional structure known as egg box, which allows the processing of hydrogels as spun or microspheres, for example. However, for dosage forms to be administered orally, such calcium alginate cross-linked systems become sensitive in basic $\mathrm{pH}$ fluids, such as intestinal regions, where the alginate matrix tends to exhibit high swell ability, delivering the drug encapsulated in its matrix abruptly [30]. To overcome this drawback, different approaches have been employed to improve the properties of the alginate matrices in an attempt to prolong the rates of release profile, such as the use of other hydrophilic macromolecules able to establish ionic interactions with carboxyl groups, such as pectin, in order to create a coating that renders the alginate system more resistant for oral administration [31].

On the other hand, studies revealed that such matrices may present certain drawbacks such as high degree of swelling, low encapsulation efficiency, and high hydrophilicity [30]. An alternative proposed was the use of other biomacromolecules with reduced hydrophilicity or even insoluble in water. In such biopolymeric associations, the second biopolymer is incorporated into the alginate gel, forming a homogeneous blend with improved physicochemical properties compared to those of pure alginate matrix. In this sense, in vitro studies have shown that the formation of a complex between alginate carboxyl groups and amine groups from zein (a hydrophobic protein) [32] made it possible to develop a prolonged release system of drugs in solutions that simulate intestinal fluid (pH 6.8 and 7.4) [33].

Less studied as DDS than polysaccharides, proteins are promising candidates for the development of innovative pharmaceutical systems. The literature reported that gelatin microparticles can serve as vehicles for the delivery of large bioactive molecules, while nanoparticles are more suitable for intravenous administration or drug delivery to the brain [34]. Gelatin hydrogels can encapsulate drugs of interest between the empty spaces formed by the biopolymer crosslinking, allowing these active molecules to diffuse into the bloodstream, such as the creation of bioinspired gelatinbased films, which incorporated antibiotic and analgesic drugs [34-36].

As exposed, the application of biopolymers in dosage forms is highly innovative, versatile, and extensive, since various polymeric combinations can be developed using the most distinct synthesis strategies. It is important to note that these systems may have limitations such as great affinity between the drug and the matrix, hindering the latter's delivery into the medium of interest, poor miscibility between biopolymers of distinct hydrophilicity, rendering the system heterogeneous and creating drug domains in the prepared device, and structural similarity, for example, in a polysaccharide blend, which makes it difficult to elucidate 
TABLE 1: Blended polymers as pharmaceutical forms for DDS discussed here.

\begin{tabular}{lccc}
\hline Polymeric blend & Form & Drug & Ref. \\
\hline PLGA-PEG & Micelle & Doxorubicin & {$[4]$} \\
Chitosan-alginate & Beads & BSA & {$[16]$} \\
Chitin-Pluronic F108 & Microparticles & Paclitaxel & {$[20]$} \\
Chitosan-gelatin & Hydrogel & Chloramphenicol & {$[22]$} \\
Chitosan-alginate & Microcapsules & Nitrofurantoin & {$[25]$} \\
Chitosan-glucomannan & Hydrogel & Ofloxacin & {$[26]$} \\
Chitosan-silk fibroin & Film & Theophylline & {$[27]$} \\
Chitosan-silk fibroin & Film & Sodium diclofenac & {$[27]$} \\
Chitosan-silk fibroin & Film & Amoxicillin & {$[27]$} \\
Chitosan-silk fibroin & Film & Salicylic acid & {$[27]$} \\
Alginate-gelatin & Film & Ciproflaxin & {$[29]$} \\
Alginate-zein & Beads & Ibuprofen & {$[32]$} \\
Chitosan-pectin & Beads & 5-Aminosalicylate & {$[33]$} \\
PEG-gelatin & Nanoparticles & Ibuprofen & {$[34]$} \\
PEG-gelatin & Hydrogel & Ciproflaxin & {$[34]$} \\
PLGA-gelatin & Nanofiber & Fenbufen & {$[34]$} \\
\hline
\end{tabular}

details on the molecular interactions between them. Therefore, biopolymers can be applied as drug delivery matrices, conferring important physical and mechanical properties to the systems, and their major limitations may be circumvented when combined with other materials. Table 1 summarized the above-mentioned DDS based on blended polymers.

\section{Biohybrid Sustained Release Systems}

A challenging strategy is the development of complex systems able to combine the properties of different materials into a single formulation with optimized properties. Such systems self-organize synergistically and can be used for the most varied applications. Although the use of the synergism among materials may suggest an innovative field of research, such approach was already employed since Ancient Greece [1,2]. An example of a hybrid system with synergistic properties is adobe, a building material composed of a mixture of clay and straw, used as an effective hybrid system in the manufacture of bricks and walls in arid regions throughout history. The addition of straw contributed significantly to preventing the formation of cracks in building structures, generating a system with superior properties by the synergism between the components [37]. There are also biohybrid systems perfectly engendered in nature, such as mother-of-pearl, formed by the interaction of calcium carbonate (aragonite) crystals strictly oriented with fibrous proteins (Lustrin A), which provides exceptional mechanical properties to the mineral. Mother-ofpearl is an inspiration for several hybrid devices of biomedical interest [9].

Organic-organic biohybrid systems have combined properties that offer many advantages compared to current DDS systems [38]. Different types of interactions such as hydrophobic, electrostatic, hydrogen bonds, donor-receptor, coordinating, and, less commonly, covalent interactions govern the molecular organization of these systems [39]. In all cases, the resulting materials must present biocompatibility and the ability to self-organize, maintaining a state of thermodynamic equilibrium [40].

3.1. Emulsions. An emulsion is a colloidal system consisting of two immiscible liquids that form small and dispersed droplets, mainly used as a strategy for the delivery of lipophilic molecules in the pharmaceutical and food industries [41]. They can be either oil-in-water $(\mathrm{O} / \mathrm{W})$ or waterin-oil (W/O) and are classified into five types: $\mathrm{O} / \mathrm{W}$ or W/O microemulsions, with droplet size between 0.1 and $5 \mu \mathrm{m}$; nanoemulsions, with droplet size generally around 20-100 nm; micellar emulsions, with droplet size ranging from 5 to $50 \mathrm{~nm}$; and double or multiple emulsions of the water-in-oil-in-water $(\mathrm{W} / \mathrm{O} / \mathrm{W})$ and oil-in-water-in-oil (O/W/O) types [41]. Despite these differences, a limitation of emulsions is the thermodynamic instability, which can result in cream formation, flocculation, coalescence, phase inversion, and "Ostwald ripening" [42]. The preparation of stable formulations requires the incorporation of emulsifiers, thickeners, gelling agents, and/or ripening inhibitors [43]. These agents stabilize the system and also confer other interesting features such as improved mechanical property. They are able to change in the final pharmaceutical form, as a biphasic gel, known as "emulgel," which is widely explored. Emulgel has an internal nonpolar liquid phase and a viscous matrix composing the continuous phase [43].

The emulgel development contributed to the preparation of novel biohybrid emulsions composed of organic excipients, combining suitable properties of all compounds. In the development of biohybrid emulsions, it is important to analyze the rheology properties and, consequently, the delivery kinetics of the compound of interest, which would be modified [44]. In general, an increase in viscosity causes a decrease in the rate of drug delivery [45]; it also affects the structural excipients organization in the final pharmaceutical form [46].

Jacobs et al. formulated emulsions modified of different concentrations of xanthan gum aiming at the delivery of acyclovir and ketoconazole for the treatment of epidermal fungal disorders [47]. Two different forms were produced from the same matrix: a cream with $0.5 \%(\mathrm{w} / \mathrm{w})$ xanthan gum and emulgel with $1.5 \%(\mathrm{w} / \mathrm{w})$ xanthan gum. The authors evaluated the physicochemical properties (viscosity, $\mathrm{pH}$, mass loss, and particle size) for 6 months. In all the analyzed parameters, the formulations had better results than those shown by the commercial form analyzed, as in the viscosity measures over time, showing the advantages of the xanthan gum use in the biohybrid system. In addition, for such systems, the in vitro permeation through human skin (obtained from abdominal plastic surgery) was sustained up to $12 \mathrm{~h}$, reaching deeper layers of the skin compared to the commercially available formulations. Wakhet et al. [48] studied the structural elucidation of hydrogel and emulgel formulations composed of agar and gelatin as the biopolymeric counterpart. X-ray diffraction (XRD) measurements confirmed the incorporation of the oil phase in the gel through the relative increase in its crystallinity, corroborated by Fourier Transform Infrared spectroscopy (FTIR-ATR) analysis, followed by the electrical 
impedance and mechanical measurements, which were superior to those of the conventional hydrogel.

Thus, the development of biohybrid emulsions can be an interesting alternative to improve the delivery of drugs, with several options of composition, and may even improve some properties of the commercially available formulations. The great advantage of these systems is the ability to solubilize hydrophobic molecules with efficiency, improving the physicochemical stability with a sustained release profile of the therapeutic molecules.

3.2. Polymer-Protein Nanoparticles Formulations. The conjugation of a polymer with a protein results in a new biomacromolecule with different physicochemical properties. These changes are usually reflected in solubility, stability, in vitro activity, biodistribution, and pharmacokinetic and pharmacodynamic profiles, as well as immunogenicity and toxicity [49]. As therapeutic agents, peptides and proteins are generally covalently bound to polymers to form peptide/proteinpolymer conjugates. Such systems have been widely studied as possible devices for drug delivery over the last decades [50].

The first polymer-protein complex was reported in 1970 when polyethylene glycol (PEG) was conjugated with bovine serum albumin (BSA) [51], opening way for a new field of study for proteins. US Food and Drug Administration (FDA) approved the first PEG-protein conjugate for routine clinical use in the early 1980s. PEG-adenosine deaminase (Adagen ${ }^{\circledR}$ ) [52] is currently used for the treatment of the severe immunodeficiency associated diseases and PEGasparaginase $\left(\right.$ Oncaspar $\left.^{\circledR}\right)$ [53] is applied in the treatment of acute lymphoblastic leukemia [54]. Conventionally, polymers are conjugated with proteins in the lysine or cysteine domains. Normally, the protein acts as an active component, while the polymer plays the role of carrier, targeting moiety or having cofunctional groups [49]. As mentioned, proteins are versatile biopolymers and their functional properties depend on their amino acid composition, besides several functional groups $\left(-\mathrm{NH}_{2},-\mathrm{COOH}\right.$, and $\left.-\mathrm{SH}\right)$, which may be used for covalent and noncovalent bonds with other molecules of interest. So far, only linear polymers such as PEG, polysaccharides (e.g., dextran), polyglutamic acid, N-(2-hydroxypropyl) methacrylamide acid, and their copolymers have been clinically explored [50]. Covalent conjugation strategies are often used in reactions of amine groups with carboxylic acid, via carbodiimide, or amine-aldehyde addition-elimination reactions, leading to nonselective conjugations and thiolene and azide-alkyne click reactions, which cause stereospecific conjugations. Noncovalent conjugations have been used as a "layer-by-layer" strategy, usually through electrostatic interactions, hydrophobic attractions, or highly selective bioaffinity bonds. Although it is common for conjugated proteins to lose their biological activity after nonspecific covalent conjugation with polymer particles, noncovalent conjugation strategies using electrostatic interactions can be adopted, which do not affect the biological activity of the native protein [55].

The work by Ge et al. [56] reported a new DDS based on self-assembled nanoparticles of BSA conjugated with polymethyl methacrylate (PMMA). PMMA is a thermoplastic material approved by FDA for medical applications. Uniform spherical nanoparticles with BSA-PMMA core and layers were prepared by precipitation to encapsulate camptothecin. The camptothecin-loaded BSA-PMMA nanoparticles showed antitumor activity improvement in both in vitro and in vivo studies, reducing the tumor growth around $79 \%$, being more effective than free drug. Zhang et al. described another biohybrid polymer-protein system as a delivery vector for nonviral genes [57]. Nanoparticles of BSApoly(dimethylamino)ethyl methacrylate (nBSA) were synthesized by in situ polymerization, with BSA as the macroinitiator. The size and surface charge of the hybrid nanoparticles (polyplexes) were controlled by modulation of the polymerization time. It was postulated that the delivery of these complexes to the cells would be facilitated by the positive charge on the surface of the polyplexes. The ability of nBSA to mediate nonviral gene delivery to cells was studied and compared to that of traditional cationic polymers, that is, polyethylenimine (PEI) and poly(2-(dimethylamino)ethyl methacrylate) (PDMA), linear and branched. The nBSA/pDNA conjugates were able to transfer genes to cells with similar or improved efficiency compared to the PEI and PDMA polymers. It is believed that the use of polymer-protein conjugates for gene delivery provides additional biofunctionality to the systems [57].

Saarai et al. [58] tested a polymer-protein nanoparticles system consisting of diblock copolymers composed of elastinlike polypeptides (ELP) bonded to FKBP12 (intracellular protein) to deliver a potent immunosuppressant, rapamycin (Rapa), and evaluated its effects on lacrimal gland inflammation in nonobese diabetic (NOD) rats. The formulations were characterized regarding purity, hydrodynamic diameter, encapsulation, and drug delivery. The dosage form associated with Rapa was successful, with a half-life five times longer $(62.5 \mathrm{~h})$ than the free drug. When administered by injection in NOD, the formulations significantly suppressed lymphocytic infiltration in the lacrimal gland compared to the control group, while reducing toxicity, proving to be a promising tool for the delivery of Rapa in the treatment of Sjögren's syndrome.

3.3. Cyclodextrins. Since 1970, biotechnological advances allowed the purification and production of cyclodextrins to be applied as pharmaceutical excipients [59]. Cyclodextrins (CD) are cyclic oligosaccharides composed of glucopyranose units, joined by 1.4 alpha linkages [60]. CD are capable of interacting noncovalently with a wide variety of lipophilic and hydrophilic molecules by forming molecular inclusion complexes in the inner (nonpolar) cavity of their macrocyclic ring or through hydrogen interactions with the hydroxyls of their outer (polar) surface [61]. Natural CD $(\alpha, \beta, \gamma$, with six, seven, and eight units of glucopyranose, resp.) underwent chemical modifications, producing even more soluble and less toxic derivatives, such as hydroxypropyl$\beta-C D$, sulfobutyl ether- $\beta-C D$, and methyl- $\beta-C D$, the first two cited as inactive pharmaceutical ingredients by the FDA. There are currently more than $35 \mathrm{CD}$-based products commercially available [62]. 
$\mathrm{CD} /$ polymer biohybrid systems were synthesized to obtain more biocompatible and multifunctional formulations. The CD are covalently linked to the backbone chain or conjugated with the side groups of polymers. When CD are synthesized by charged functional monomers, electrostatic interactions may occur, allowing the spontaneous formation of nanospheres, nanogels, and nanocapsules [63]. El Fagui et al. described the development of CD (hydrophilic)-PLA (hydrophobic) nanoparticulated biohybrid systems. Structural characterization by NMR (nuclear magnetic resonance) and small-angle X-ray scattering (SAXS) demonstrated that the CD coated the PLA nanoparticles by absorption, protecting their hydrophobic core which modified the physicochemical properties of the PLA nanoparticles, opening the possibility for future conjugations [64]. Another interesting possibility is the incorporation of CD in hydrogels matrices, stabilizing the inclusion complexes formed by CD in their hydrophilic network. In addition, the presence of CD optimizes mechanical properties, makes the system sensitive to physiological stimuli, and allows the sustained delivery of drugs [65].

Zhou et al. described the creation of an injectable thermosensitive gel composed of chitosan and $\beta$-CD, which presented a lamellar structure with fractal characteristics and showed a prolonged release profile for Aspirin ${ }^{\circledR}$. It was evidenced that the presence of CD in the system modulated the drug release profile, delivering around 50\% after $24 \mathrm{~h}$ [66]. McCormack and Gregoriadis were the first to propose the encapsulation of drug-CD inclusion complex by liposomes, improving its ability to encapsulate hydrophobic drugs with success. Thus, the complex formed was encapsulated in the internal aqueous medium of the liposomes, which maintained the integrity of the lipid bilayer after inclusion of CD. Therefore, this approach has been widely explored as DDS [67]. Recently, Sharma et al. developed a CD-liposome inclusion complex with improved physicochemical stability for the transdermal delivery of aceclofenac (ACE), an antiinflammatory drug indicated for the osteoarthritis treatment. The drug was previously conjugated with a $\beta$-CD and the resulting compound was encapsulated by multivesicular liposomes. FTIR analysis suggested that Van der Waals interactions governed the ACE- $\beta$-CD system, as well as this complex conjugated to liposomes. The resulting material was further incorporated in a polymeric matrix and processed as a gel, which presented better in vitro permeation (through mouse skin) compared to the control group, being a promising candidate for the treatment of osteoarthritis [68].

3.4. Liposomes. Liposomes are composed of lipid bilayers, in which amphiphilic phospholipids carry a hydrophilic group (head) and two hydrophobic acyl chains (tail) [69]. In these lipid vesicles, formed by the hydrophobic effect to maximize the interaction between the acyl chains, protecting them from the contact with the aqueous medium, the hydrophobic tails turn towards the inside of the lamella and the polar heads are exposed outward, in contact with water [69]. Due to the presence of an aqueous compartment and lipid bilayers, liposomes can incorporate hydrophilic and hydrophobic molecules, which is quite interesting for a drug carrier [70].
Several advances are required for liposomes to be clinically applied, given their short half-life and low physicochemical stability [71]. One of the strategies to improve these limitations is the association of liposomes with other drug delivery systems, usually polymers, forming the liposomebased biohybrid systems.

To improve the stability and half-life of liposomes in the bloodstream, a hydrophilic polymer is commonly used, usually PEG, covalently bound to a phospholipid, for example, phosphatidylethanolamine (PE). In these so-called stealth liposomes, even containing a low molar fraction of PEG$\mathrm{PE}$ (usually 5\%), the surface of the liposomes is coated by PEG, creating a steric hindrance that reduces the absorption of plasma protein and, consequently, the metabolism [72]. This strategy has been widely used to produce stealth liposome with the particle size in the range of $100 \mathrm{~nm}$. Abe et al. evaluated, by NMR, the molecular state of PEG on the surface of liposomes composed of DSPC (distearoyl-3phosphatidylcholine), finding that the PEG chain's flexibility depends on its molecular weight and lipid composition [73]. However, extensive PEGylation can also cause inhibition of cellular uptake, which is not desired for the cancer treatment. Pozzi et al. reported by SAXS that phase separation on liposome can occur due to PEG. Thus, they developed a moderated PEGylated (size $2 \mathrm{~K}$ ) multicomponent liposome that hindered protein adsorption but still presented high cellular uptake in cancer cells [74]. Therefore, all of these studies were relevant for the development of efficient biohybrid liposomal pharmaceutical forms, contributing to the elucidation of their performance in the bloodstream [75, 76].

Another interesting approach was the combination of liposomes with (bio)polymers as matrices for hydrogels preparation. The association of liposomes of different charges (negative, neutral, and positive) with a chitosan hydrogel was evaluated. Using rhodamine as a model molecule, fluorescence experiments revealed its in vitro delivery profile from the hybrid system. The hydrogel containing anionic liposomes showed a burst release of rhodamine, probably because the positive charges of chitosan destabilized the anionic liposomal membrane, which was able to increase the rhodamine delivery. The neutral liposomes/chitosan preparation presented a more sustained delivery profile than the anionic liposomes. On the other hand, the cationic liposomes/chitosan preparation, in turn, presented the most sustained rhodamine profile of all liposomal hydrogels tested. The positive charges of the phospholipids and chitosan chains probably were repelled, preserving the integrity of the lipid bilayer [77]. Another study developed a liposomal thermosensitive hydrogel for the delivery of doxorubicin. In these forms, besides the in vitro sustained delivery profile, a decrease in in vivo toxicity was observed [78]. On the other hand, Caddeo et al. showed the use of chitosan as coating for liposomes containing quercetin. The formulation was able to modulate the quercetin release profile according to the $\mathrm{pH}$, increasing the bioavailability of the encapsulated drug. Chitosan was able to protect quercetin-loaded liposomes and provide special resistance to acidic environments, such as that found in gastric environment [79]. 
Liposomes can also be used to target active molecules to specific sites, such as tumors. The incorporation of different ligands, such as monoclonal antibodies, peptides, growth factors, or integrin, improves the specificity in the drug release. Protein-coated liposomes are considered promising in comparison to conventional liposomes. Different forces such as electrostatic interactions, or even more specific such as receptor-ligand interactions, will determine the biohybrid system interaction with the plasma membrane of cells. A biohybrid system composed of liposomes and specific proteins (MCF-7) for the transport of DOX through the bloodbrain barrier (BBB) was recently described. Through covalent interactions between the liposomal lipid and protein, the system was proven to be effective in overcoming BBB, showed a sustained delivery profile, and was able to kill tumor cells in vitro [80].

3.5. Innovative Hybrid Lipid Nanoparticles: Solid Lipid Nanoparticles (SLN), Nanostructured Lipid Carriers (NLC), Lipospheres, and Lipid Nanocapsules. Lipid nanoparticles are innovative nanocarriers that have been studied since 1990, representing a progress in the physicochemical stability of the lipid-based formulations and the encapsulation of hydrophobic molecules. Solid lipid nanoparticles (SLN), the first generation of these systems, are composed of solid lipids (core) at room temperature, surfactant, and water. Nanostructured lipid carriers (NLC) are the second generation, having as the lipid phase a blend of one or more solid and liquid lipids at room temperature [81]. Both systems still present some disadvantages, such as a possible expulsion of the drug over time (SLN) and low capacity to encapsulate hydrophilic molecules [82] due to limited thermodynamic interactions between the hydrophilic and hydrophobic interfaces of the drug and the lipid nanoparticle, respectively [83].

An important strategy employed to minimize such limitations of SLN and NLC is their hybridization with other classes of biomaterials, mainly polymers. The electrostatic and hydrogen interactions among the excipients thus provide biohybrid systems with improved properties, such as the delivery profile of active molecules increasing plasma halflife [83], permeation [84], and efficacy [85]. Furthermore, this combination of materials can be used to modulate viscosity, consistency, and mucoadhesion of lipid nanoparticles [86] or to add thermoreversible properties to the systems $[87,88]$.

Pandit and Dash [88] modified the surface of SLN systems in order to improve the encapsulation of the hydrophilic antineoplastic ifosfamide (IFO), indicated for the treatment of soft tissue sarcoma, for oral administration. The formulations were composed of glycerol monooleate, oleic acid (surfactant), and cross-linked chitosan. Despite the low encapsulation efficiency of IFO $(\sim 10 \%)$, the biohybrid SLN showed an in vitro sustained drug delivery profile up to $60 \mathrm{~h}$. The system was able to protect drug against degradation in acidic environments, demonstrating the benefits of using hybrid systems in a single dosage form.

Biohybrid hydrogels (Figure 1) have been widely employed in association with SLN for topical applications. Among the most commonly used polymeric matrices are

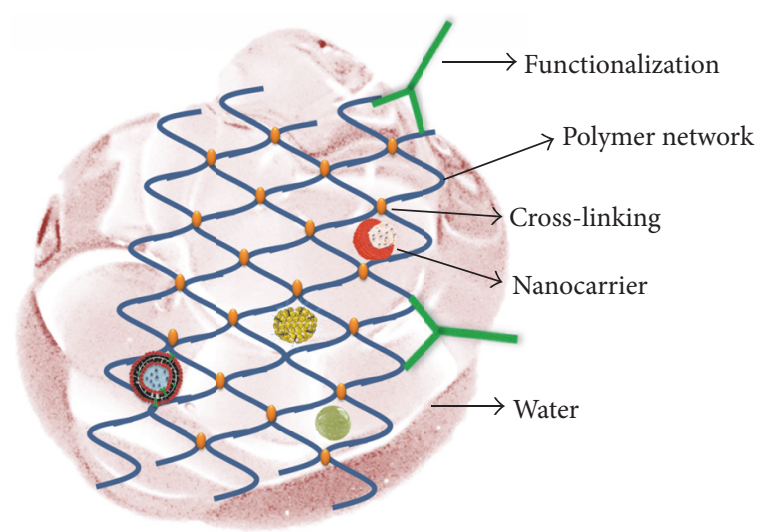

FIGURE 1: Hydrogels are semisolid pharmaceutical forms composed mostly of water, tridimensional polymeric network, and crosslinked chains. Their hybridization process allows incorporating other functional groups, drug-loaded nanocarriers, and triggers, improving the DDS performance.

Carbopol [89, 90], alginate [91], and dextran [92]. Hao et al. [93] developed a thermosensitive biohybrid hydrogel composed of poloxamer to improve the ophthalmic drug delivery. The hydrogel gelled only in contact with the ocular mucosa, improving adhesion and permeation of the Chinese phytochemical resin, known as "Dragon's blood." The SLN with particle size around $150 \mathrm{~nm}$ showed an abrupt increase when incorporated in the hydrogel $(\sim 450 \mathrm{~nm})$ due to the adsorption of poloxamer portions on the surface of the nanoparticles. The biohybrid hydrogels improved the stability of the drug and increased mucoadhesion and penetration capacity in the cornea when in vitro evaluated. The evaluation of transcorneal mechanism across the cornea was performed in rabbits, showing that the system allowed a prolonged residence time and reduced the systemic side effect. On the other hand, Hazzah et al. [86] developed biohybrid sponges based on hydroxypropyl methylcellulose (HPMC) and polycarbophil as polymeric matrices, incorporating curcumin-loaded SLN for oral mucosal administration. The resulting materials showed a sustained delivery profile of curcumin and excellent mucoadhesion properties evaluated in five adult healthy volunteers.

The incorporation of polyelectrolytes on the surface of nanoparticles is also a strategy to improve stability and optimize targeting. Doktorovová et al. [94] developed PEGylated-based NLC through the blend between Precirol (solid lipid) and a PEGylated liquid lipid (Labrasol) for the sustained delivery of fluticasone propionate in dermatology. The nanoparticles showed excellent encapsulation efficiency (95\%) and physicochemical stability (60 days), stored at room temperature. The crystallinity and polymorphism of the compounds were analyzed by wide-angle X-ray scattering (WAXS) and DSC, which showed that although the formulations presented a lower melting point than pure Precirol $\left(63^{\circ} \mathrm{C}\right)$, the nanoparticles were in solid state at room temperature, with a melting point above $50^{\circ} \mathrm{C}$, as required for biological applications.

Devkar et al. [95] developed a biohybrid NLC based on glycerol monostearate and Capryol 90 as the lipid matrix, 
stabilized with soy lecithin and poloxamer and superficially modified by the natural Delonix regia gum, combining mucoadhesive properties to the system. The hybrid system was intended for intranasal delivery of ondansetron and showed ca. $44 \%$ encapsulation efficiency. Mucoadhesive properties were achieved and the drug efficiently reached the target site (brain) with a sustained release profile, as observed by in vivo biodistribution assay, showing higher drug concentration in the brain than the plasma compared to intravenous route. Wong et al. described polymer-lipid hybrid nanoparticle systems composed of SLN and soybeanbased anionic polymer, which efficiently loaded doxorubicin $(\sim 50 \%)$ and improved the toxicity against human breast cancer cells [96]. Li and coworkers designed polymer-lipid hybrid nanoparticles based on SLN and dextran sulfate sodium as a polymeric counterpart to complex the ionized verapamil. The structural characterization suggested that verapamil was in the amorphous form into SLN, further corroborated by the high encapsulation efficiency $(\sim 88 \%)$, when the lipid : drug ratio in the hybrid was 1:1(w/w) [97].

Rose et al. [87] developed optimized lipid-polymer nanoparticles composed of PLGA electrostatically modified with dimethyl octadecyl ammonium as cationic surfactant and conjugated with a complex formed between the glycolipid trehalose dibehenate and PVA. This formulation aimed to act as an immunopotentiator to modify cellular and humoral immunity by antibodies and Th1/Th17 responses, proposed as adjuvant in topical vaccine, improving the specificity of the system against many bacterial pathogens of the oral mucosa. The main force that governed its molecular arrangement was the electrostatic interaction between the quaternary ammonium group of dimethyl octadecyl ammonium and the negatively charged PLGA-PVA polymer conjugate. The lipid polymer nanoparticles were able to stimulate immune response, acting as efficient adjuvants, as demonstrated by in vivo mice immunization model.

On the other hand, lipospheres are drug upload systems composed of an oily solid core (triglycerides) [98], in which a hydrophobic drug may be dissolved or dispersed, stabilized by a monolayer of phospholipid molecules that cover its surface [99]. These systems were developed for the delivery of bioactive compounds by parenteral and topical routes, exhibiting a great in vivo anti-inflammatory effect by piroxicam-loaded lipospheres [100]. They consist of solid microparticles with sizes between 0.2 and $100 \mu \mathrm{m}$ [101]. Their advantages over other DDS (emulsions, liposomes, and polymer nanoparticles) include physical stability, being easy to disperse in aqueous medium, simple preparation, high retention of hydrophobic drugs in the core, and prolonged delivery [102]. Lipospheres have been used for the delivery of several classes of drugs, such as local anesthetics [99], anti-inflammatories [100], antineoplastic [101], immunosuppressant [102], hypoglycemic agents [103], antimalarial agents [104], and antihypertensive agents [105].

Lipospheres are usually associated with other types of carrier systems, such as cyclodextrins or polymeric matrices. These biohybrid systems aim to potentiate the lipospheres' properties and/or modulate drug delivery. An example is the incorporation of butyl methoxydibenzoylmethane
(BMDBM)/hydroxypropyl- $\beta$-cyclodextrin (HP- $\beta$-CD) complex into lipospheres, aiming to increase the effect and photostability of the sunscreen agent. DSC analysis revealed that the endothermic peak of the pure drug disappeared in the liposphere-BMDBM/HP- $\beta$-CD sample. Such thermal behavior suggested that the drug was dispersed in the liposphere core, in an amorphous state, which explained the sustained drug release, as well as the increase in photostability achieved [106]. In another study, Ma et al. [107] developed a biohybrid system based on PLGA copolymer conjugated with the liposphere core for the delivery BSA for oral administration. The lipospheres exhibited spherical morphology with PLGA hydrophobic core delimited by a weak ring, suggesting that the lipid layer was on its surface, as shown by scanning electron microscopy. This system, compared to conventional PLGA microspheres, showed better uploading of BSA ( 91\%) and a sustained delivery profile. The biohybrid system improved the protein delivery capacity as well as its bioavailability, being potentially useful for oral administration.

Lastly, lipid nanocapsules are further bioinspired hybrid nanocarriers, composed of a triglycerides core delimited by a polymeric shell, stabilized by surfactants and/or cosurfactants, and obtained by thermal inversion [108]. Recently, Jones et al. developed lipid nanocapsules functionalized with cytotoxic T-cells (CTLs) as mediator of antigen recognitiontriggered drug release. In an in vivo model of HIV infection (mice), the nanocapsules were able to detect immunosuppressed cells with a sustained interleukin-15 (IL-15) release at the HIV-specific site [109]. Kim et al. proposed a treatment for non-small cell lung cancer, based on the release of erlotinib encapsulated by PEGylated polypeptide-lipid nanocapsules. The nanocapsules were biocompatible with particle size lower than $200 \mathrm{~nm}$ and a monodisperse distribution. The PEG covering exerted a protective role, acting as a molecular barrier that prolonged the erlotinib release in the acidic target medium. The lipid nanocapsules were found cytotoxic for NCI-H358 and HCC-827 lung cancer cells. The efficacy was evaluated in mice by a tumor regression profile model, showing an improved therapeutic action for the hybrid lipid nanocapsules in comparison with free drug, being a promise for the treatment of lung cancer [110]. Antonow et al. described lipid nanocapsules loaded doxorubicin to study the in vitro antiproliferative property and determine the nanocapsules uptake by MCF-7 cells. The nanocapsules' particle size was $c a .200 \mathrm{~nm}$ and a high antiproliferative capacity $(>90 \%)$ was observed. These systems were cytotoxic for MCF-7 cell exhibiting a sustained effect evaluated for $72 \mathrm{~h}$ [111]. Quercetin lipid nanocapsules for dermatological uses were prepared by phase inversion method. The formulations presented particle size around $50 \mathrm{~nm}$, high encapsulation efficiency, and antioxidant activity (92\%) in vitro, with a sustained release profile over $24 \mathrm{~h}$, being stable for 28 days at room temperature [112]. While lipid nanocapsules appear as a novel hybrid-based approach with great potential as DDS, allowing for small-sized $(<100 \mathrm{~nm})$ nanoparticles' preparation, cytotoxic issues related to their high surfactant content are still pending. Figure 2 compares the structure of some types of traditional DDS and the respective hybrid systems. Table 2 shows relevant information of main published works 
TABLE 2: Main published works regarding biohybrid systems for sustained drug delivery, listed in terms of kind, activity, and encapsulation efficiency (EE\%).

\begin{tabular}{|c|c|c|c|}
\hline Biohybrid system & $\begin{array}{c}\text { Therapeutic } \\
\text { molecule }\end{array}$ & $\mathrm{EE} \%$ & Ref. \\
\hline NE-alginate/chitosan & Capsaicin & $68.0 \%$ & [44] \\
\hline Emulgel-jojoba oil/HPMC & Clotrimazole & - & [46] \\
\hline Emulgel-xanthan gum & $\begin{array}{l}\text { Acyclovir and } \\
\text { ketoconazole }\end{array}$ & - & {$[47]$} \\
\hline Emulgel-lecithin soy & Ketoprofen & - & [113] \\
\hline Nanogel-agar & Sodium diclofenac & - & {$[48]$} \\
\hline Emulgel-CMC & Sodium diclofenac & - & {$[114]$} \\
\hline Emulgel-gelatin & Metronidazole & - & [45] \\
\hline Poly(SMA)-neocarzinostatin & Neocarzinostatin & - & [115] \\
\hline PEG-DNA & DNA & - & {$[52]$} \\
\hline PEG-streptavidin & Streptavidin & - & [116] \\
\hline PEG-L-asparaginase & L-Asparaginase & - & {$[53]$} \\
\hline PEG-streptavidin & Streptavidin & - & [117] \\
\hline PDMA-BSA & DNA & - & {$[57]$} \\
\hline PMMA-BSA & Camptothecin & $11.0 \%$ & [56] \\
\hline ELP-FKBP & Rapamycin & - & {$[58]$} \\
\hline Liposome-chitosan & Doxorubicin & $98.0 \%$ & [78] \\
\hline Liposome-cellulose & Quercetin & $40.0 \%$ & {$[77]$} \\
\hline Liposome-cellulose & Rutin & $58.0 \%$ & {$[77]$} \\
\hline Liposome-gel & Lidocaine & $21.6 \%$ & [118] \\
\hline Liposome-gel & Bupivacaine & $98.8 \%$ & [119] \\
\hline Liposome-alginate & Benzocaine & $63.2 \%$ & [120] \\
\hline Liposome-PEG & Lidocaine & $98.8 \%$ & {$[73]$} \\
\hline Cyclodextrin/liposome & Quercetin & $91.0 \%$ & {$[121]$} \\
\hline Cyclodextrin/liposome & Tretinoin & $88.7 \%$ & {$[122]$} \\
\hline Cyclodextrin/liposome & Curcumin & $50.0 \%$ & [123] \\
\hline Cyclodextrin/PLGA & Oxaprozin & $62.0 \%$ & [124] \\
\hline SLN-PLGA-PEG-PLGA & $\begin{array}{c}2- \\
\text { Methoxyestradiol }\end{array}$ & $91.3 \%$ & [125] \\
\hline SLN-hydrogel & Natural resin & - & [93] \\
\hline SLN-polycarbophil & Curcumin & $88.1 \%$ & [86] \\
\hline SLN-B ${ }_{12}$ vitamin & Insulin & $55.9 \%$ & [85] \\
\hline SLN-chitosan & Amphotericin B & $88.5 \%$ & {$[126]$} \\
\hline SLN-PLGA & Flurbiprofen & $91.7 \%$ & {$[127]$} \\
\hline SLN-dicetyl phosphate & Retinyl palmitate & $99.1 \%$ & [128] \\
\hline SLN-PEG & EGFP Plasmid & $89.0 \%$ & [129] \\
\hline SLN-PEG & Paclitaxel & $11.0 \%$ & [130] \\
\hline SLN-PEG & $\begin{array}{l}\text { Salbutamol } \\
\text { sulphate }\end{array}$ & $30.0 \%$ & [131] \\
\hline SLN-PEG & Noscapine & $83.6 \%$ & {$[84]$} \\
\hline SLN-chitosan & Carbamazepine & $66.7 \%$ & [132] \\
\hline SLN-chitosan & Ifosfamide & - & {$[89]$} \\
\hline SLN-dextran & Ibuprofen & $99.1 \%$ & [133] \\
\hline SLN-chitosan & Tretinoin & $99.6 \%$ & [134] \\
\hline SLN-PLGA & DNA & $93.1 \%$ & [135] \\
\hline
\end{tabular}

TABLE 2: Continued.

\begin{tabular}{lcrc}
\hline Biohybrid system & Therapeutic molecule & EE\% & Ref. \\
\hline NLC-natural gum & Ondansetron & $29.9 \%$ & {$[95]$} \\
(D. regia) & Fluticasone propionate & $97.0 \%$ & {$[94]$} \\
NLC-PEG & Docetaxel & $77.5 \%$ & {$[136]$} \\
NLC-peptides & Biochanin A & $99.0 \%$ & {$[137]$} \\
NLC-PEG & Curcumin & $96.6 \%$ & {$[138]$} \\
NLC-chitosan & Fubiprofen & $97.5 \%$ & {$[139]$} \\
NLC-chitosan & Butyl & $85.0 \%$ & {$[106]$} \\
Lipospheres- $\beta$-CD & methoxydibenzoylmethane & & {$[140]$} \\
Lipospheres- $\beta$-CD & Rifampicin & - & {$[107]$} \\
Lipospheres-PLGA & Albumin & $90.8 \%$ & {$[141]$} \\
Lipospheres-PLGA & Donepezil hydrochloride & - & {$[142]$} \\
Lipospheres-PEG & Ceftriaxone & $60.1 \%$ & {$[142]$} \\
Lipospheres-PEG & Gentamicin & $85.0 \%$ & {$[143]$} \\
Lipid nanocapsules & Interleukin-15 (IL-15) & - & {$[109]$} \\
Lipid nanocapsules & Erlotinib & $90.0 \%$ & {$[110]$} \\
Lipid nanocapsules & Doxorubicin & $90.0 \%$ & {$[111]$} \\
Lipid nanocapsules & Quercetin & $92.0 \%$ & {$[112]$} \\
\hline
\end{tabular}

related to biohybrid systems for sustained delivery of therapeutic molecules.

\section{In Vivo Performance of Biohybrid Polymer-Based Hybrid DDS}

The polymer-based hybrid systems' development has been exponentially increased in the last years. However, most of the publications noticed the in vitro performance of such materials in addition to their structural elucidation. This theoretical approach is fundamental to guarantee their success and physicochemical stability. Therefore, multidisciplinary efforts are necessary to precisely evaluate the efficacy, bioavailability, and pharmacokinetics of these multipurpose systems, considering that there is a lack of animal models for testing different DDS for specific applications [144].

In general, we observed advantages of such hybrid systems over their respective traditional DDS, which significantly improved the in vivo pharmacokinetics and the activity of several classes of drugs [67, 72, 100, 104, 118, 119, 128]. As in the case of protein nanoparticles, where polymer-protein conjugates for gene delivery provided biofunctionality to the systems [57], other formulations reduced in vivo tumor growth [56]; and the anti-inflammatory activity five times higher than the traditional protein nanoparticle was noticed [58]. Regarding the hybrid lipid-based DDS, the outlook is exciting. The well-known stealth liposomes commonly hybridized with PEG were able to improve the in vivo short half-life [71] and the low bloodstream stability [72] of pure liposomes in mice. The decrease of in vivo chemotherapeutics toxicity was noticed for liposomes in gel [78]. With respect to hybrid lipid nanocapsules, a sustained interleukin-15 (IL15) release at the HIV-specific site in an HIV infection 

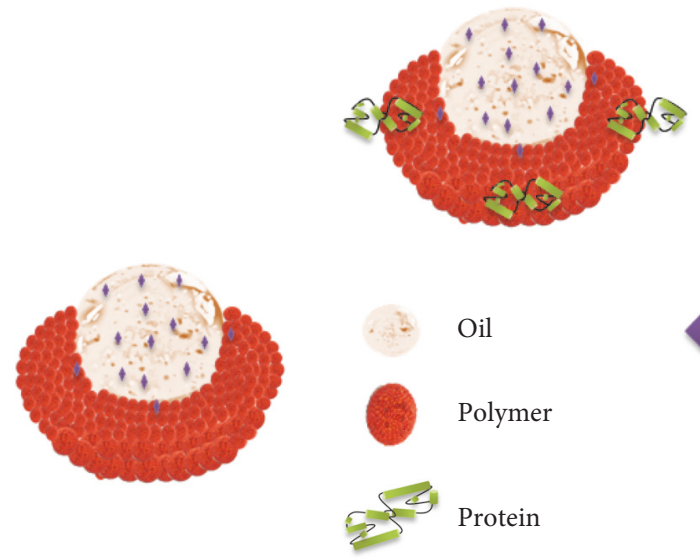

Oil
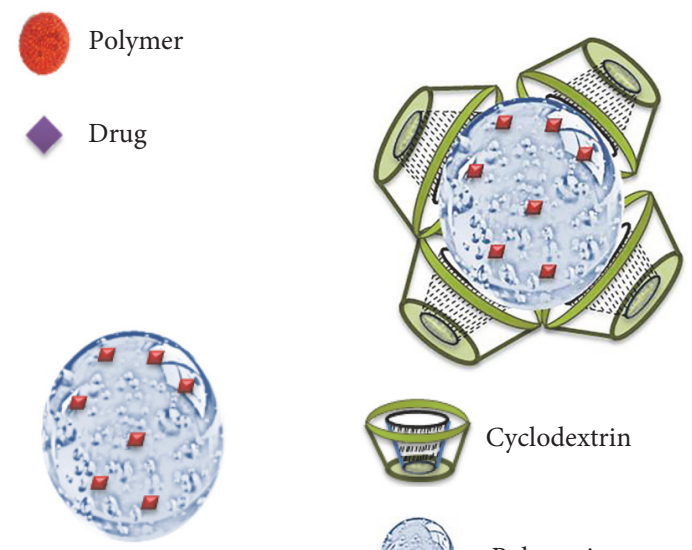

Polymeric core

Polymeric core

$$
\square \quad \text { Drug }
$$

Drug
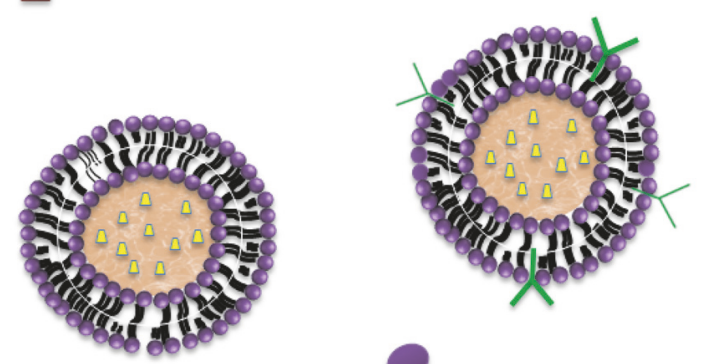

(\} Amphiphilic lipid

?) Amphiphilic lipid

Drug

Drug

Y PEG

(a)

(b)

FIGURE 2: Schematic representation of some traditional nanocarriers' structures (a) and their respective organic biohybrid systems (b). 
model in mice was provided [109]. A promising hybridbased lipid nanocapsule was described for the treatment of lung cancer, which described the in vivo (mice) tumor regression profile [110]. In vivo pharmacokinetics studies in rats demonstrated higher bioavailability of hybrid artesunateloaded lipospheres when compared to the commercial tablet for malaria treatment [104]. Different in vivo studies were described for SLN- and NLC-based hybrid systems for topical and parenteral applications, using mice, rat, and rabbit, as well as in human clinical trial, which demonstrated the effectiveness, nontoxicity, and bioavailability of these biodevices compared to their respective traditional DDS or free drug $[86,88,94,96]$.

\section{Perspectives}

It is utopian to expect that a single nanosystem will accomplish all drug delivery demands. Despite the progress in pharmaceutical nanotechnology, all current DDS present advantages and limitations for its clinical use. The DDS composed of biopolymers as single component can exhibit undesirable mechanical properties and do not have the advantages of drug encapsulation. On the other hand, despite the capability of encapsulation of hydrophilic molecules, protein nanoparticles and liposomes can present low physicochemical stability, as also occurs in the emulsions, affecting their therapeutic properties. SLN and NLC present longterm stability and low encapsulation efficiency of hydrophilic molecules. CD and all the colloidal formulations cannot be applied for topical routes with success, due to undesirable adhesion, spreadability, and viscosity, which are advantages of the emulsions and polymeric systems. Finally, CD and liposomal systems are able to incorporate both hydrophilic and hydrophobic molecules in their structure. Considering that there is no perfect DDS system, a deep understanding of the structural, physicochemical, and biological properties of the route and drug is necessary, making the choice of the appropriate device possible.

Therefore, the strategic association of different organic biomaterials combines suitable intrinsic properties of each component in a single DDS. The organic-organic biohybrid systems represent important advances in drug delivery field, such as efficient encapsulation of hydrophilic molecules by SLN and NLC formulations; improved mechanical (emulsions), adhesion (colloidal formulations and CD), and physicochemical properties (emulsions, liposomes, and protein nanoparticles); functionalization of protein and lipid nanocarriers enhancing target specificity and cancer cells cytotoxicity; improved protein bioavailability for oral administration; multiple therapeutic properties in a single system; and better in vivo performance than the most of conventional DDS.

The use of (bio)polymeric excipients in biohybrid systems development is especially relevant due to its diversity, abundance, low cost, and biocompatibility. These versatile materials have several uses, such as surface modifiers, complexing agents, stimulus-response dependence, and matrices for solid pharmaceutical forms that improve mechanical, adhesion, and permeation properties of the nanocarriers.
This class of biohybrid systems (organic-organic) deserves attention, considering the ability to interact with ability to interact with site of interest, predicting and controlling the DDS performance when in contact with the target, solving several limitations of traditional DDS.

There are several hybridization processes of DDS currently employed to improve the nanocarrier specificity to the targets. Changes in the global charge of the traditional nanocarriers by superficial functionalization or thiomerization provide hybrid smart drug delivery systems, which are able to electrostatically interact with the different mucosal tissues, fixing them at the desired site. Moreover, molecular designed polymer-based hybrid DDS can combine several stimuli-dependent effects in a single biodevice, such as $\mathrm{pH}$ and temperature, which should be specific to the action site, as a tumor tissue. Therefore, a multidisciplinary point of view is mandatory to understand the particularities of the target, carrier, and therapeutic molecules, linking physical, chemical, and biological aspects to design creative and efficient systems to improve the efficacy and stability of delivered drugs.

However, despite the excellent performance in the in vitro and in vivo assays, the human trials of these creative DDS are still scarce, which hinders the market arrival of the biohybrid nanocarriers systems. Along with that, the regulatory issues of the excipients safety as well as their conjugation in a single nanostructured biodevice are not fully established, contributing to the delay in commercial availability of these promising DDS. Moreover, the long-term consequences of nanostructured systems as DDS in the environment have not been fully elucidated yet.

\section{Conclusions}

The development of biohybrid advanced materials for sustained release has been widely described over the last years. However, a thorough review of these findings is not yet available in the literature. Characteristically, a synergistic interaction among the components, combining the advantages of each raw material, governed the molecular organization of these systems. Different systems have been efficiently prepared, which combine different classes of organic excipients, such as polymer, protein, and lipid, yielding formulations or pharmaceutical forms with several advantages, such as dynamic interaction with targets, enhancing their performance. This work revised the biohybrid DDS, elucidating their advantages and limitations, contributing to further study of creative, molecularly planned, and safe biohybrid systems as advanced materials for the sustained release of active molecules. These systems showed exciting in vitro and in vivo results, being a promise for the delivery of many drugs for different routes. This work also directed efforts to inspire new research with this innovator multidisciplinary approach.

\section{Abbreviations}

BMDBM: Butyl methoxydibenzoylmethane

BSA: $\quad$ Bovine serum albumin

CD: Cyclodextrin 
CMC: Carboxymethyl cellulose

DDS: Drug delivery systems

DOX: Doxorubicin

DSC: Differential scanning calorimetry

ELP: $\quad$ Elastomer-like proteins/polypeptides

FKBP12: Protein receptor for rapamycin

FTIR: Fourier Transform Infrared spectroscopy

HA: Hyaluronic acid

NLC: Nanostructured lipid carriers

NMR: Nuclear magnetic resonance

PEG: Polyethylene glycol

PEI: Polyethylenimine

PLA: Polylactide

PLGA: Polylactide-co-glycolide

PDMA: Poly(dimethylamino)ethyl methacrylate

PMMA: Polymethyl methacrylate

PVA: Polyvinyl acid

PVP: Polyvinylpyrrolidone

Ref.: References

SLN: $\quad$ Solid lipid nanoparticles

XRD: X-ray diffraction.

\section{Conflicts of Interest}

The authors declare that there are no conflicts of interest regarding the publication of this paper.

\section{Authors' Contributions}

All the authors contributed equally to this work.

\section{Acknowledgments}

The authors would like to acknowledge FAPESP (no. 14/25372-0 and no. 14/14457-5), CNPq (productivity grant, Eneida de Paula), and FAPEMA (Brazil-MA) project UNIVERSAL 01118/16 for funding the research and the Espaço da Escrita, Coordenadoria Geral da Universidade, UNICAMP, for the language services provided.

\section{References}

[1] S. Ramakrishna, J. Mayer, E. Wintermantel, and K. W. Leong, "Biomedical applications of polymer-composite materials: a review," Composites Science and Technology, vol. 61, no. 9, pp. 1189-1224, 2001.

[2] K. A. Dubey, C. V. Chaudhari, Y. K. Bhardwaj, and L. Varshney, "Polymers, blends and nanocomposites for implants, scaffolds and controlled drug release applications," Advanced Structured Materials, vol. 66, pp. 1-44, 2017.

[3] A. Z. Wilczewska, K. Niemirowicz, K. H. Markiewicz, and H. Car, "Nanoparticles as drug delivery systems," Pharmacological Reports, vol. 64, no. 5, pp. 1020-1037, 2012.

[4] M. Joglekar and B. G. Trewyn, "Polymer-based stimuliresponsive nanosystems for biomedical applications," Biotechnology Journal, vol. 8, no. 8, pp. 931-945, 2013.

[5] C. Puglia and F. Bonina, "Lipid nanoparticles as novel delivery systems for cosmetics and dermal pharmaceuticals," Expert Opinion on Drug Delivery, vol. 9, no. 4, pp. 429-441, 2012.
[6] A. A. Attama, M. A. Momoh, and P. F. Builders, "Lipid nanoparticulate drug delivery systems: A revolution in dosage form design and development," in Recent Advances in Novel Drug Carrier Systems, pp. 107-140, Intech, 2012.

[7] T. Loftsson and D. Duchene, "Cyclodextrins and their pharmaceutical applications," International Journal of Pharmaceutics, vol. 329, no. 1-2, pp. 1-11, 2007.

[8] A. S. Hoffman, "The origins and evolution of "controlled" drug delivery systems," Journal of Controlled Release, vol. 132, no. 3, pp. 153-163, 2008.

[9] A. Aghabegi Moghanjoughi, D. Khoshnevis, and A. Zarrabi, "A concise review on smart polymers for controlled drug release," Drug Delivery and Translational Research, vol. 6, no. 3, pp. 333340, 2016.

[10] E. Ruiz-Hitzky, M. Darder, and P. Aranda, "Functional biopolymer nanocomposites based on layered solids," Journal of Materials Chemistry, vol. 15, no. 35-36, pp. 3650-3662, 2005.

[11] Z. Liu, Y. Jiao, Y. Wang, C. Zhou, and Z. Zhang, "Polysaccharides-based nanoparticles as drug delivery systems," Advanced Drug Delivery Reviews, vol. 60, no. 15, pp. 1650-1662, 2008.

[12] A. Sionkowska, M. Wisniewski, J. Skopinska, C. J. Kennedy, and T. J. Wess, "Molecular interactions in collagen and chitosan blends," Biomaterials, vol. 25, no. 5, pp. 795-801, 2004.

[13] X.-G. Chen, C.-S. Liu, C.-G. Liu, X.-H. Meng, C. M. Lee, and H.J. Park, "Preparation and biocompatibility of chitosan microcarriers as biomaterial," Biochemical Engineering Journal, vol. 27, no. 3, pp. 269-274, 2006.

[14] F.-C. Wu, R.-L. Tseng, and R.-S. Juang, "A review and experimental verification of using chitosan and its derivatives as adsorbents for selected heavy metals," Journal of Environmental Management, vol. 91, no. 4, pp. 798-806, 2010.

[15] H. Sashiwa and S.-I. Aiba, "Chemically modified chitin and chitosan as biomaterials," Progress in Polymer Science, vol. 29, no. 9, pp. 887-908, 2004.

[16] Y. Xu, C. Zhan, L. Fan, L. Wang, and H. Zheng, "Preparation of dual crosslinked alginate-chitosan blend gel beads and in vitro controlled release in oral site-specific drug delivery system," International Journal of Pharmaceutics, vol. 336, no. 2, pp. 329337, 2007.

[17] A. Matalanis, O. G. Jones, and D. J. McClements, "Structured biopolymer-based delivery systems for encapsulation, protection, and release of lipophilic compounds," Food Hydrocolloids, vol. 25, no. 8, pp. 1865-1880, 2011.

[18] C. Alvarez-Lorenzo, B. Blanco-Fernandez, A. M. Puga, and A. Concheiro, "Crosslinked ionic polysaccharides for stimulisensitive drug delivery," Advanced Drug Delivery Reviews, vol. 65, no. 9, pp. 1148-1171, 2013.

[19] V. Rana, P. Rai, A. K. Tiwary, R. S. Singh, J. F. Kennedy, and C. J. Knill, "Modified gums: Approaches and applications in drug delivery," Carbohydrate Polymers, vol. 83, no. 3, pp. 1031-1047, 2011.

[20] V. R. Sinha, A. K. Singla, S. Wadhawan et al., "Chitosan microspheres as a potential carrier for drugs," International Journal of Pharmaceutics, vol. 274, no. 1-2, pp. 1-33, 2004.

[21] J. Berger, M. Reist, J. M. Mayer, O. Felt, N. A. Peppas, and R. Gurny, "Structure and interactions in covalently and ionically crosslinked chitosan hydrogels for biomedical applications," European Journal of Pharmaceutics and Biopharmaceutics, vol. 57, no. 1, pp. 19-34, 2004.

[22] X. Z. Shu and K. J. Zhu, "Chitosan/gelatin microspheres prepared by modified emulsification and ionotropic gelation," Journal of Microencapsulation, vol. 18, no. 2, pp. 237-245, 2001. 
[23] V. L. Gonçalves, M. C. Laranjeira, V. T. Fávere, and R. C. Pedrosa, "Effect of crosslinking agents on chitosan microspheres in controlled release of diclofenac sodium," Polímeros: Ciência e Tecnologia, vol. 15, no. 1, pp. 6-12, 2005.

[24] H. K. Stulzer, L. Lacerda, M. P. Tagliari, M. A. S. Silva, V. T. Fávere, and M. C. M. Laranjeira, "Synthesis and characterization of cross-linked malonylchitosan microspheres for controlled release of acyclovir," Carbohydrate Polymers, vol. 73, no. 3, pp. 490-497, 2008.

[25] P. R. Hari, T. Chandy, and C. P. Sharma, "Chitosan/calcium alginate microcapsules for intestinal delivery of nitrofurantoin," Journal of Microencapsulation, vol. 13, no. 3, pp. 319-329, 1996.

[26] H. Yu, J. Lu, and C. Xiao, "Preparation and properties of novel hydrogels from oxidized konjac glucomannan cross-linked chitosan for in vitro drug delivery," Macromolecular Bioscience, vol. 7, no. 9-10, pp. 1100-1111, 2007.

[27] R. Rujiravanit, S. Kruaykitanon, A. M. Jamieson, and S. Tokura, "Preparation of Crosslinked Chitosan/Silk Fibroin Blend Films for Drug Delivery System," Macromolecular Bioscience, vol. 3, no. 10, pp. 604-611, 2003.

[28] M. U. Adikwu and C. O. Esimone, "Biopolymers in drug delivery: Recent advances and challenges," Biopolymers in Drug Delivery: Recent Advances and Challenges, 2009.

[29] Z. Dong, Q. Wang, and Y. Du, "Alginate/gelatin blend films and their properties for drug controlled release," Journal of Membrane Science, vol. 280, no. 1-2, pp. 37-44, 2006.

[30] K. Y. Lee and D. J. Mooney, "Alginate: properties and biomedical applications," Progress in Polymer Science, vol. 37, no. 1, pp. 106126, 2012.

[31] T. Coviello, P. Matricardi, C. Marianecci, and F. Alhaique, "Polysaccharide hydrogels for modified release formulations," Journal of Controlled Release, vol. 119, no. 1, pp. 5-24, 2007.

[32] A. C. S. Alcântara, P. Aranda, M. Darder, and E. RuizHitzky, "Bionanocomposites based on alginate-zein/layered double hydroxide materials as drug delivery systems," Journal of Materials Chemistry, vol. 20, no. 42, pp. 9495-9504, 2010.

[33] L. N. M. Ribeiro, A. C. S. Alcântara, M. Darder, P. Aranda, F. M. Araújo-Moreira, and E. Ruiz-Hitzky, "Pectin-coated chitosanLDH bionanocomposite beads as potential systems for colontargeted drug delivery," International Journal of Pharmaceutics, vol. 463, no. 1, pp. 1-9, 2014.

[34] M. Foox and M. Zilberman, "Drug delivery from gelatin-based systems," Expert Opinion on Drug Delivery, vol. 12, no. 9, pp. 1547-1563, 2015.

[35] S. Nagarajan, L. Soussan, M. Bechelany et al., "Novel biocompatible electrospun gelatin fiber mats with antibiotic drug delivery properties," Journal of Materials Chemistry B, vol. 4, no. 6, pp. 1134-1141, 2016.

[36] V. DiTizio, C. Karlgard, L. Lilge, A. E. Khoury, M. W. Mittelman, and F. DiCosmo, "Localized drug delivery using crosslinked gelatin gels containing liposomes: Factors influencing liposome stability and drug release," Journal of Biomedical Materials Research Part B: Applied Biomaterials, vol. 51, no. 1, pp. 96-106, 2000.

[37] P. Gomez-Romero, "Hybrid organic-inorganic materials - in search of synergic activity," Advanced Materials, vol. 13, no. 3, pp. 163-174, 2001.

[38] L. N. M. Ribeiro, A. C. S. Alcântara, M. Darder et al., "Bionanocomposites containing magnetic graphite as potential systems for drug delivery," International Journal of Pharmaceutics, vol. 477, no. 1-2, pp. 553-563, 2014.
[39] M. R. Dreher, A. J. Simnick, K. Fischer et al., “Temperature triggered self-assembly of polypeptides into multivalent spherical micelles," Journal of the American Chemical Society, vol. 130, no. 2, pp. 687-694, 2008.

[40] S. Rangelov and S. Pispas, Polymer and Polymer-Hybrid Nanoparticles: From Synthesis to Biomedical Applications, 2014.

[41] M. J. Lawrence and G. D. Rees, "Microemulsion-based media as novel drug delivery systems," Advanced Drug Delivery Reviews, vol. 45, no. 1, pp. 89-121, 2000.

[42] T. F. Tadros, "Emulsion Formation and Stability," Emulsion Formation and Stability, 2013.

[43] K. P. Mohammed Haneefa, S. Easo, P. V. Hafsa, G. P. Mohanta, and C. Nayar, "characterization and Optimization of oromucosal Clotrimazole Emulgel formulation," Journal of Pharmaceutical Sciences, vol. 5, pp. 249-254, 2013.

[44] A.-J. Choi, C.-J. Kim, Y.-J. Cho, J.-K. Hwang, and C.-T. Kim, "Characterization of Capsaicin-Loaded Nanoemulsions Stabilized with Alginate and Chitosan by Self-assembly," Food and Bioprocess Technology, vol. 4, no. 6, pp. 1119-1126, 2011.

[45] Ajazuddin, A. Alexander, A. Khichariya et al., "Recent expansions in an emergent novel drug delivery technology: Emulgel," Journal of Controlled Release, vol. 171, no. 2, pp. 122-132, 2013.

[46] M. Shahin, S. Abdel Hady, M. Hammad, and N. Mortada, "Novel jojoba oil-based emulsion gel formulations for clotrimazole delivery," AAPS PharmSciTech, vol. 12, no. 1, pp. 239-247, 2011.

[47] G. A. Jacobs, M. Gerber, M. M. Malan, J. L. du Preez, L. T. Fox, and J. du Plessis, "Topical delivery of acyclovir and ketoconazole," Drug Delivery, vol. 23, no. 2, pp. 631-641, 2016.

[48] S. Wakhet, V. K. Singh, S. Sahoo et al., "Characterization of gelatin-agar based phase separated hydrogel, emulgel and bigel: a comparative study," Journal of Materials Science: Materials in Medicine, vol. 26, no. 2, 2015.

[49] W. Zhao, F. Liu, Y. Chen, J. Bai, and W. Gao, "Synthesis of welldefined protein-polymer conjugates for biomedicine," Polymer (United Kingdom), vol. 66, Article ID 17726, pp. A1-A10, 2015.

[50] B. Mukherjee, S. D. Karmakar, C. M. Hossain, and S. Bhattacharya, "Peptides, proteins and peptide/protein-polymer conjugates as drug delivery system," Protein and Peptide Letters, vol. 21, no. 11, pp. 1121-1128, 2014.

[51] A. Abuchowski, T. Vanes, N. C. Palczuk, and F. F. Davis, "Alteration of immunological properties of bovine serum albumin by covalent attachment of polyethylene glycol," The Journal of Biological Chemistry, vol. 252, no. 11, pp. 3578-3581, 1977.

[52] Y. Levy, M. S. Hershfield, C. Fernandez-Mejia et al., "Adenosine deaminase deficiency with late onset of recurrent infections: response to treatment with polyethylene glycol-modified adenosine deaminase," Journal of Pediatrics, vol. 113, no. 2, pp. 312-317, 1988.

[53] M. L. Graham, "Pegaspargase: a review of clinical studies," Advanced Drug Delivery Reviews, vol. 55, no. 10, pp. 1293-1302, 2003.

[54] R. Duncan, H. Ringsdorf, and R. Satchi-Fainaro, "Polymer therapeutics-Polymers as drugs, drug and protein conjugates and gene delivery systems: Past, present and future opportunities," Journal of Drug Targeting, vol. 14, no. 6, pp. 337-341, 2006.

[55] C. Boyer, X. Huang, M. R. Whittaker, V. Bulmus, and T. P. Davis, "An overview of protein-polymer particles," Soft Matter, vol. 7, no. 5, pp. 1599-1614, 2011. 
[56] J. Ge, E. Neofytou, J. Lei, R. E. Beygui, and R. N. Zare, "Proteinpolymer hybrid nanoparticles for drug delivery," Small, vol. 8, no. 23, pp. 3573-3578, 2012.

[57] J. Zhang, Y. Lei, A. Dhaliwal et al., "Protein-polymer nanoparticles for nonviral gene delivery," Biomacromolecules, vol. 12, no. 4, pp. 1006-1014, 2011.

[58] A. Saarai, V. Kasparkova, T. Sedlacek, and P. Saha, "On the development and characterisation of crosslinked sodium alginate/gelatine hydrogels," Journal of the Mechanical Behavior of Biomedical Materials, vol. 18, pp. 152-166, 2013.

[59] P. J. Sicard and M. H. Saniez, "Biosynthesis of cycloglycosyl transferase and obtention of its enzymatic reaction products," in Duchene D (ed) Cyclodextrins and their industrial use, D. Duchene, Ed., pp. 75-103, Editions de Santé, Paris, France, 1987.

[60] E. M. M. del Valle, "Cyclodextrins and their uses: a review," Process Biochemistry, vol. 39, no. 9, pp. 1033-1046, 2004.

[61] R. Challa, A. Ahuja, J. Ali, and R. K. Khar, "Cyclodextrins in drug delivery: an updated review," AAPS PharmSciTech, vol. 6, no. 2, pp. E329-E357, 2005.

[62] S. V. Kurkov and T. Loftsson, "Cyclodextrins," International Journal of Pharmaceutics, vol. 453, no. 1, pp. 167-180, 2013.

[63] J. Zhang and P. X. Ma, "Cyclodextrin-based supramolecular systems for drug delivery: Recent progress and future perspective," Advanced Drug Delivery Reviews, vol. 65, no. 9, pp. 1215-1233, 2013.

[64] A. El Fagui, F. Dalmas, C. Lorthioir, V. Wintgens, G. Volet, and C. Amiel, "Well-defined core-shell nanoparticles containing cyclodextrin in the shell: A comprehensive study," Polymer Journal, vol. 52, no. 17, pp. 3752-3761, 2011.

[65] A. Concheiro and C. Alvarez-Lorenzo, "Chemically crosslinked and grafted cyclodextrin hydrogels: From nanostructures to drug-eluting medical devices," Advanced Drug Delivery Reviews, vol. 65, no. 9, pp. 1188-1203, 2013.

[66] H. Y. Zhou, Z. Y. Wang, X. Y. Duan et al., "Design and evaluation of chitosan- $\beta$-cyclodextrin based thermosensitive hydrogel," Biochemical Engineering Journal, vol. 111, pp. 100-107, 2016.

[67] B. McCormack and G. Gregoriadis, "Drugs-in-cyclodextrinsin liposomes: a novel concept in drug delivery," International Journal of Pharmaceutics, vol. 112, no. 3, pp. 249-258, 1994.

[68] G. Sharma, M. Kaur, K. Raza, K. Thakur, and O. P. Katare, "Aceclofenac- $\beta$-cyclodextrin-vesicles: A dual carrier approach for skin with enhanced stability, efficacy and dermatokinetic profile," RSC Advances, vol. 6, no. 25, pp. 20713-20727, 2016.

[69] A. Joset, A. Grammenos, M. Hoebeke, and B. Leyh, "Smallangle neutron scattering investigation of cholesterol-doped DMPC liposomes interacting with $\beta$-cyclodextrin," Journal of Inclusion Phenomena and Macrocyclic Chemistry, vol. 84, no. 12, pp. 153-161, 2015.

[70] E. De Paula, C. M. Cereda, L. F. Fraceto et al., "Micro and nanosystems for delivering local anesthetics," Expert Opinion on Drug Delivery, vol. 9, no. 12, pp. 1505-1524, 2012.

[71] B. S. Pattni, V. V. Chupin, and V. P. Torchilin, "New Developments in Liposomal Drug Delivery," Chemical Reviews, vol. 115, no. 19, pp. 10938-10966, 2015.

[72] J. Gubernator, "Active methods of drug loading into liposomes: Recent strategies for stable drug entrapment and increased in vivo activity," Expert Opinion on Drug Delivery, vol. 8, no. 5, pp. 565-580, 2011.

[73] K. Abe, K. Higashi, K. Watabe et al., "Effects of the PEG molecular weight of a PEG-lipid and cholesterol on PEG chain flexibility on liposome surfaces," Colloids and Surfaces A:
Physicochemical and Engineering Aspects, vol. 474, pp. 63-70, 2015.

[74] D. Pozzi, V. Colapicchioni, G. Caracciolo et al., "Effect of polyethyleneglycol (PEG) chain length on the bio-nano- interactions between PEGylated lipid nanoparticles and biological fluids: From nanostructure to uptake in cancer cells," Nanoscale, vol. 6, no. 5, pp. 2782-2792, 2014.

[75] Z. Varga, A. Wacha, U. Vainio, J. Gummel, and A. Bóta, “Characterization of the PEG layer of sterically stabilized liposomes: a SAXS study," Chemistry and Physics of Lipids, vol. 165, no. 4, pp. 387-392, 2012.

[76] J. Hurler, S. Žakelj, J. Mravljak et al., "The effect of lipid composition and liposome size on the release properties of liposomes-in-hydrogel," International Journal of Pharmaceutics, vol. 456, no. 1, pp. 49-57, 2013.

[77] S. N. Park, M. H. Lee, S. J. Kim, and E. R. Yu, "Preparation of quercetin and rutin-loaded ceramide liposomes and drugreleasing effect in liposome-in-hydrogel complex system," Biochemical and Biophysical Research Communications, vol. 435, no. 3, pp. 361-366, 2013.

[78] S. Ren, Y. Dai, C. Li et al., "Pharmacokinetics and pharmacodynamics evaluation of a thermosensitive chitosan based hydrogel containing liposomal doxorubicin," European Journal of Pharmaceutical Sciences, vol. 92, pp. 137-145, 2016.

[79] C. Caddeo, O. Díez-Sales, R. Pons et al., "Cross-linked chitosan/liposome hybrid system for the intestinal delivery of quercetin," Journal of Colloid and Interface Science, vol. 461, pp. 69-78, 2016.

[80] M. Sánchez-Purrà, V. Ramos, V. A. Petrenko, V. P. Torchilin, and S. Borrós, "Double-targeted polymersomes and liposomes for multiple barrier crossing," International Journal of Pharmaceutics, vol. 511, no. 2, pp. 946-956, 2016.

[81] L. N. M. Ribeiro, M. Franz-Montan, M. C. Breitkreitz et al., "Nanostructured lipid carriers as robust systems for topical lidocaine-prilocaine release in dentistry," European Journal of Pharmaceutical Sciences, vol. 93, pp. 192-202, 2016.

[82] L. N. M. Ribeiro, M. C. Breitkreitz, V. A. Guilherme, Rodrigues da Silva G. H., and V. M. Couto, "Natural lipids-based NLC: from pre-formulation to in vivo studies," European Journal of Pharmaceutical Sciences, vol. 106, pp. 102-112, 2017.

[83] V. Kumar and R. K. Prud'homme, "Thermodynamic limits on drug loading in nanoparticle cores," Journal of Pharmaceutical Sciences, vol. 97, no. 11, pp. 4904-4914, 2008.

[84] J. Madan, R. S. Pandey, V. Jain, O. P. Katare, R. Chandra, and A. Katyal, "Poly (ethylene)-glycol conjugated solid lipid nanoparticles of noscapine improve biological half-life, brain delivery and efficacy in glioblastoma cells," Nanomedicine: Nanotechnology, Biology and Medicine, vol. 9, no. 4, pp. 492-503, 2013.

[85] H. He, P. Wang, C. Cai, R. Yang, and X. Tang, "Gel-core SLN containing insulin: Another way to improve oral absorption," International Journal of Pharmaceutics, vol. 493, pp. 451-459.

[86] H. A. Hazzah, R. M. Farid, M. M. A. Nasra, M. A. El-Massik, and O. Y. Abdallah, "Lyophilized sponges loaded with curcumin solid lipid nanoparticles for buccal delivery: Development and characterization," International Journal of Pharmaceutics, vol. 492, no. 1-2, Article ID 14974, pp. 248-257, 2015.

[87] F. Rose, J. E. Wern, P. T. Ingvarsson et al., "Engineering of a novel adjuvant based on lipid-polymer hybrid nanoparticles: a quality-by-design approach," Journal of Controlled Release, vol. 210, pp. 48-57, 2015. 
[88] A. A. Pandit and A. K. Dash, "Surface-modified solid lipid nanoparticulate formulation for ifosfamide: development and characterization.," Nanomedicine (London, England), vol. 6, no. 8, pp. 1397-1412, 2011.

[89] A. Garg and S. Singh, "Targeting of eugenol-loaded solid lipid nanoparticles to the epidermal layer of human skin," Nanomedicine, vol. 9, no. 8, pp. 1223-1238, 2014.

[90] X. Chen, L.-H. Peng, Y.-H. Shan et al., "Astragaloside IV-loaded nanoparticle-enriched hydrogel induces wound healing and anti-scar activity through topical delivery," International Journal of Pharmaceutics, vol. 447, no. 1-2, pp. 171-181, 2013.

[91] P. Severino, M. V. Chaud, A. Shimojo et al., "Sodium alginatecross-linked polymyxin B sulphate-loaded solid lipid nanoparticles: antibiotic resistance tests and HaCat and NIH/3T3 cell viability studies," Colloids and Surfaces B: Biointerfaces, vol. 129, pp. 191-197, 2015.

[92] M. A. Casadei, F. Cerreto, S. Cesa et al., "Solid lipid nanoparticles incorporated in dextran hydrogels: A new drug delivery system for oral formulations," International Journal of Pharmaceutics, vol. 325, no. 1-2, pp. 140-146, 2006.

[93] J. Hao, X. Wang, Y. Bi et al., "Fabrication of a composite system combining solid lipid nanoparticles and thermosensitive hydrogel for challenging ophthalmic drug delivery," Colloids and Surfaces B: Biointerfaces, vol. 114, pp. 111-120, 2014.

[94] S. Doktorovová, J. Araújo, M. L. Garcia, E. Rakovský, and E. B. Souto, "Formulating fluticasone propionate in novel PEGcontaining nanostructured lipid carriers (PEG-NLC)," Colloids and Surfaces B: Biointerfaces, vol. 75, no. 2, pp. 538-542, 2010.

[95] T. B. Devkar, A. R. Tekade, and K. R. Khandelwal, "Surface engineered nanostructured lipid carriers for efficient nose to brain delivery of ondansetron $\mathrm{HCl}$ using Delonix regia gum as a natural mucoadhesive polymer," Colloids and Surfaces B: Biointerfaces, vol. 122, pp. 143-150, 2014.

[96] H. L. Wong, A. M. Rauth, R. Bendayan et al., "A new polymerlipid hybrid nanoparticle system increases cytotoxicity of doxorubicin against multidrug-resistant human breast cancer cells," Pharmaceutical Research, vol. 23, no. 7, pp. 1574-1585, 2006.

[97] Y. Li, N. Taulier, A. M. Rauth, and X. Y. Wu, "Screening of lipid carriers and characterization of drug-polymer-lipid interactions for the rational design of polymer-lipid hybrid nanoparticles (PLN)," Pharmaceutical Research, vol. 23, no. 8, pp. 18771887, 2006.

[98] R. Cortesi, E. Esposito, G. Luca, and C. Nastruzzi, "Production of lipospheres as carriers for bioactive compounds," Biomaterials, vol. 23, no. 11, pp. 2283-2294, 2002.

[99] S. Toongsuwan, L.-C. Li, B. K. Erickson, and H.-C. Chang, "Formulation and characterization of bupivacaine lipospheres," International Journal of Pharmaceutics, vol. 280, no. 1-2, pp. 5765, 2004.

[100] A. A. Attama and V. E. Mpamaugo, "Pharmacodynamics of piroxicam from self-emulsifying lipospheres formulated with homolipids extracted from Capra hircus," Drug Delivery: Journal of Delivery and Targeting of Therapeutic Agents, vol. 13, no. 2, pp. 133-137, 2006.

[101] R. Cavalli, O. Caputo, and M. R. Gasco, "Solid lipospheres of doxorubicin and idarubicin," International Journal of Pharmaceutics, vol. 89, no. 1, pp. R9-R12, 1993.

[102] T. Bekerman, J. Golenser, and A. Domb, “Cyclosporin Nanoparticulate Lipospheres for Oral Administration," Journal of Pharmaceutical Sciences, vol. 93, no. 5, pp. 1264-1270, 2004.

[103] M. Payasi, M. Bhowmick, G. K. Pandey, A. Joshi, and B. Dubey, "Development and characterization of pioglitazone loaded liposphere for the effective treatment of diabetes mellitus type 2," International Journal of Biomedical and Advance Research, vol. 4, 749 pages, 2004.

[104] E. E. Chinaeke, S. A. Chime, J. D. N. Ogbonna, A. A. Attama, C. C. Müller-Goymann, and V. C. Okore, "Evaluation of dika wax-soybean oil-based artesunate-loaded lipospheres: In vitroin vivo correlation studies," Journal of Microencapsulation, vol. 31, no. 8, pp. 796-804, 2014.

[105] S. S. Pandit and A. T. Patil, "Formulation and in vitro evaluation of buoyant controlled release lercanidipine lipospheres," Journal of Microencapsulation, vol. 26, no. 7, pp. 635-641, 2009.

[106] S. Scalia, R. Tursilli, N. Sala, and V. Iannuccelli, "Encapsulation in lipospheres of the complex between butyl methoxydibenzoylmethane and hydroxypropyl- $\beta$-cyclodextrin," International Journal of Pharmaceutics, vol. 320, no. 1-2, pp. 79-85, 2006.

[107] T. Ma, L. Wang, T. Yang, D. Wang, G. Ma, and S. Wang, "PLGAlipid liposphere as a promising platform for oral delivery of proteins," Colloids and Surfaces B: Biointerfaces, vol. 117, pp. 512519, 2014.

[108] B. Heurtault, P. Saulnier, B. Pech, J.-E. Proust, and J.-P. Benoit, "A novel phase inversion-based process for the preparation of lipid nanocarriers," Pharmaceutical Research, vol. 19, no. 6, pp. 875-880, 2002.

[109] R. B. Jones, S. Mueller, S. Kumari et al., "Antigen recognitiontriggered drug delivery mediated by nanocapsule-functionalized cytotoxic T-cells," Biomaterials, vol. 117, pp. 44-53, 2017.

[110] J. Kim, T. Ramasamy, J. Y. Choi et al., "PEGylated polypeptide lipid nanocapsules to enhance the anticancer efficacy of erlotinib in non-small cell lung cancer," Colloids and Surfaces B: Biointerfaces, vol. 150, pp. 393-401, 2017.

[111] M. B. Antonow, A. C. C. Asbahr, P. Raddatz et al., "Liquid formulation containing doxorubicin-loaded lipid-core nanocapsules: Cytotoxicity in human breast cancer cell line and in vitro uptake mechanism," Materials Science and Engineering C: Materials for Biological Applications, vol. 76, pp. 374-382, 2017.

[112] T. Hatahet, M. Morille, A. Shamseddin, A. Aubert-Pouëssel, J. M. Devoisselle, and S. Bégu, "Dermal quercetin lipid nanocapsules: Influence of the formulation on antioxidant activity and cellular protection against hydrogen peroxide," International Journal of Pharmaceutics, vol. 518, no. 1-2, pp. 167-176, 2017.

[113] R. Ambala and S. K. Vemula, "Formulation and characterization of ketoprofen emulgels," Journal of Applied Pharmaceutical Science, vol. 5, no. 7, pp. 112-117, 2015.

[114] F. A. Mohammed, "Topical permeation characteristics of diclofenac sodium from NaCMC gels in comparison with conventional gel formulations," Drug Development and Industrial Pharmacy, vol. 27, no. 10, pp. 1083-1097, 2001.

[115] H. Maeda, M. Ueda, T. Morinaga, and T. Matsumoto, "Conjugation of poly (styrene-co-maleic acid) derivatives to the antitumor protein neocarzinostatin: pronounced improvements in pharmacological properties," Journal of Medicinal Chemistry, vol. 28 , no. 4, pp. 455-461, 1985.

[116] Chen Guohua and A. S. Hoffman, "preparation and properties of thermoreversbile, phase-separating enzyme-oligo(Nisopropylacrylamide) conjugates," Bioconjugate Chemistry, vol. 4, no. 6, pp. 509-514, 1993.

[117] J. M. Hannink, J. J. Cornelissen, and J. A. Farrera, "ProteinPolymer Hybrid Amphiphiles," Angewandte Chemie International Edition, vol. 40, no. 24, pp. 4732-4734, 2001. 
[118] M. Franz-Montan, D. Baroni, G. Brunetto et al., "Liposomal lidocaine gel for topical use at the oral mucosa: Characterization, in vitro assays and in vivo anesthetic efficacy in humans," Journal of Liposome Research, vol. 25, no. 1, pp. 11-19, 2015.

[119] M. Franz-Montan, C. M. S. Cereda, A. Gaspari et al., "Liposomal-benzocaine gel formulation: Correlation between in vitro assays and in vivo topical anesthesia in volunteers," Journal of Liposome Research, vol. 23, no. 1, pp. 54-60, 2013.

[120] R. Cohen, H. Kanaan, G. J. Grant, and Y. Barenholz, "Prolonged analgesia from Bupisome and Bupigel formulations: From design and fabrication to improved stability," Journal of Controlled Release, vol. 160, no. 2, pp. 346-352, 2012.

[121] F. Maestrelli, M. L. González-Rodríguez, A. M. Rabasco, C. Ghelardini, and P. Mura, "New "drug-in cyclodextrin-in deformable liposomes" formulations to improve the therapeutic efficacy of local anaesthetics," International Journal of Pharmaceutics, vol. 395, no. 1-2, pp. 222-231, 2010.

[122] A. Ascenso, M. Cruz, C. Euletério et al., "Novel tretinoin formulations: A drug-in-cyclodextrin-in-liposome approach," Journal of Liposome Research, vol. 23, no. 3, pp. 211-219, 2013.

[123] S. S. Dhule, P. Penfornis, T. Frazier et al., "Curcumin-loaded $\gamma$-cyclodextrin liposomal nanoparticles as delivery vehicles for osteosarcoma," Nanomedicine, vol. 8, no. 4, pp. 440-451, 2012.

[124] P. Mura, F. Maestrelli, M. Cecchi, M. Bragagni, and A. Almeida, "Development of a new delivery system consisting in 'drugin cyclodextrinin PLGA nanoparticles,' Journal of Microencapsulation, vol. 27, no. 6, pp. 479-486, 2010.

[125] X. Guo, F. Cui, Y. Xing, Q. Mei, and Z. Zhang, "Investigation of a new injectable thermosensitive hydrogel loading solid lipid nanoparticles," Die Pharmazie, vol. 66, no. 12, pp. 948-952, 2011.

[126] V. Jain, A. Gupta, V. K. Pawar et al., "Chitosan-Assisted Immunotherapy for Intervention of Experimental Leishmaniasis via Amphotericin B-Loaded Solid Lipid Nanoparticles," Applied Biochemistry and Biotechnology, vol. 174, no. 4, pp. 1309-1330, 2014.

[127] S. K. Jain, M. K. Chourasia, R. Masuriha et al., "Solid lipid nanoparticles bearing flurbiprofen for transdermal delivery," Drug Delivery: Journal of Delivery and Targeting of Therapeutic Agents, vol. 12, no. 4, pp. 207-215, 2005.

[128] H. S. Jeon, J. E. Seo, M. S. Kim et al., "A retinyl palmitate-loaded solid lipid nanoparticle system: Effect of surface modification with dicetyl phosphate on skin permeation in vitro and antiwrinkle effect in vivo," International Journal of Pharmaceutics, vol. 452, no. 1-2, pp. 311-320, 2013.

[129] F. Jing, J. Li, D. Liu, C. Wang, and Z. Sui, "Dual ligands modified double targeted nano-system for liver targeted gene delivery," Pharmaceutical Biology, vol. 51, no. 5, pp. 643-649, 2013.

[130] J.-H. Kim, Y. Kim, K. H. Bae, T. G. Park, J. H. Lee, and K. Park, "Tumor-targeted delivery of paclitaxel using low density lipoprotein-mimetic solid lipid nanoparticles," Molecular Pharmaceutics, vol. 12, no. 4, pp. 1230-1241, 2015.

[131] Y. Hong, F. Q. Hu, and H. Yuan, "Effect of PEG2000 on drug delivery characterization from solid lipid nanoparticles," Die Pharmazie, vol. 61, no. 4, pp. 312-315, 2006.

[132] R. Nair, A. C. Kumar, V. K. Priya, C. M. Yadav, and P. Y. Raju, "Formulation and evaluation of chitosan solid lipid nanoparticles of carbamazepine," Lipids in Health and Disease, vol. 11, article no. 72, 2012.

[133] P. Paolicelli, F. Cerreto, S. Cesa et al., "Influence of the formulation components on the properties of the system SLNdextran hydrogel for the modified release of drugs," Journal of Microencapsulation, vol. 26, no. 4, pp. 355-364, 2009.
[134] D. M. Ridolfi, P. D. Marcato, G. Z. Justo, L. Cordi, D. Machado, and N. Durán, "Chitosan-solid lipid nanoparticles as carriers for topical delivery of tretinoin," Colloids and Surfaces B: Biointerfaces, vol. 93, pp. 36-40, 2012.

[135] L. Zhu, S. Xie, Z. Dong, X. Wang, Y. Wang, and W. Zhou, "Effects of poly(lactic-co-glycolic acid) on preparation and characteristics of plasmid DNA-loaded solid lipid nanoparticles," IET Nanobiotechnology, vol. 5, no. 3, pp. 79-85, 2011.

[136] W. Gao, B. Xiang, T.-T. Meng, F. Liu, and X.-R. Qi, "Chemotherapeutic drug delivery to cancer cells using a combination of folate targeting and tumor microenvironment-sensitive polypeptides," Biomaterials, vol. 34, no. 16, pp. 4137-4149, 2013.

[137] L. Wang, Q. Luo, T. Lin et al., "PEGylated nanostructured lipid carriers (PEG-NLC) as a novel drug delivery system for biochanin A," Drug Development and Industrial Pharmacy, vol. 41, no. 7, pp. 1204-1212, 2015.

[138] D. Liu, J. Li, H. Pan et al., "Potential advantages of a novel chitosan- $\mathrm{N}$-acetylcysteine surface modified nanostructured lipid carrier on the performance of ophthalmic delivery of curcumin," Scientific Reports, vol. 6, Article ID 28796, 2016.

[139] B. Tian, Q. Luo, S. Song et al., "Novel surface-modified nanostructured lipid carriers with partially deacetylated water-soluble chitosan for efficient ocular delivery," Journal of Pharmaceutical Sciences, vol. 101, no. 3, pp. 1040-1049, 2012.

[140] C. Singh, L. V. S. K. Koduri, V. Dhawale et al., "Potential of aerosolized rifampicin lipospheres for modulation of pulmonary pharmacokinetics and bio-distribution," International Journal of Pharmaceutics, vol. 495, no. 2, pp. 627-632, 2015.

[141] S. A. Yehia, A. H. Elshafeey, and I. Elsayed, "Biodegradable donepezil lipospheres for depot injection: Optimization and invivo evaluation," Journal of Pharmacy and Pharmacology, vol. 64, no. 10, pp. 1425-1437, 2012.

[142] A. A. Attama, C. E. Okafor, P. F. Builders, and O. Okorie, "Formulation and in vitro evaluation of a PEGylated microscopic lipospheres delivery system for ceftriaxone sodium," Drug Delivery, vol. 16, no. 8, pp. 448-457, 2009.

[143] M. A. Momoh and C. O. Esimone, "Phospholipon 90H (P90H)Based PEGylated microscopic lipospheres delivery system for gentamicin: An antibiotic evaluation," Asian Pacific Journal of Tropical Biomedicine, vol. 2, no. 11, pp. 889-894, 2012.

[144] M. Franz-Montan, L. N. Ribeiro, M. C. Volpato et al., "Recent advances and perspectives in topical oral anesthesia," Expert Opinion on Drug Delivery, vol. 1, pp. 673-684, 2016. 

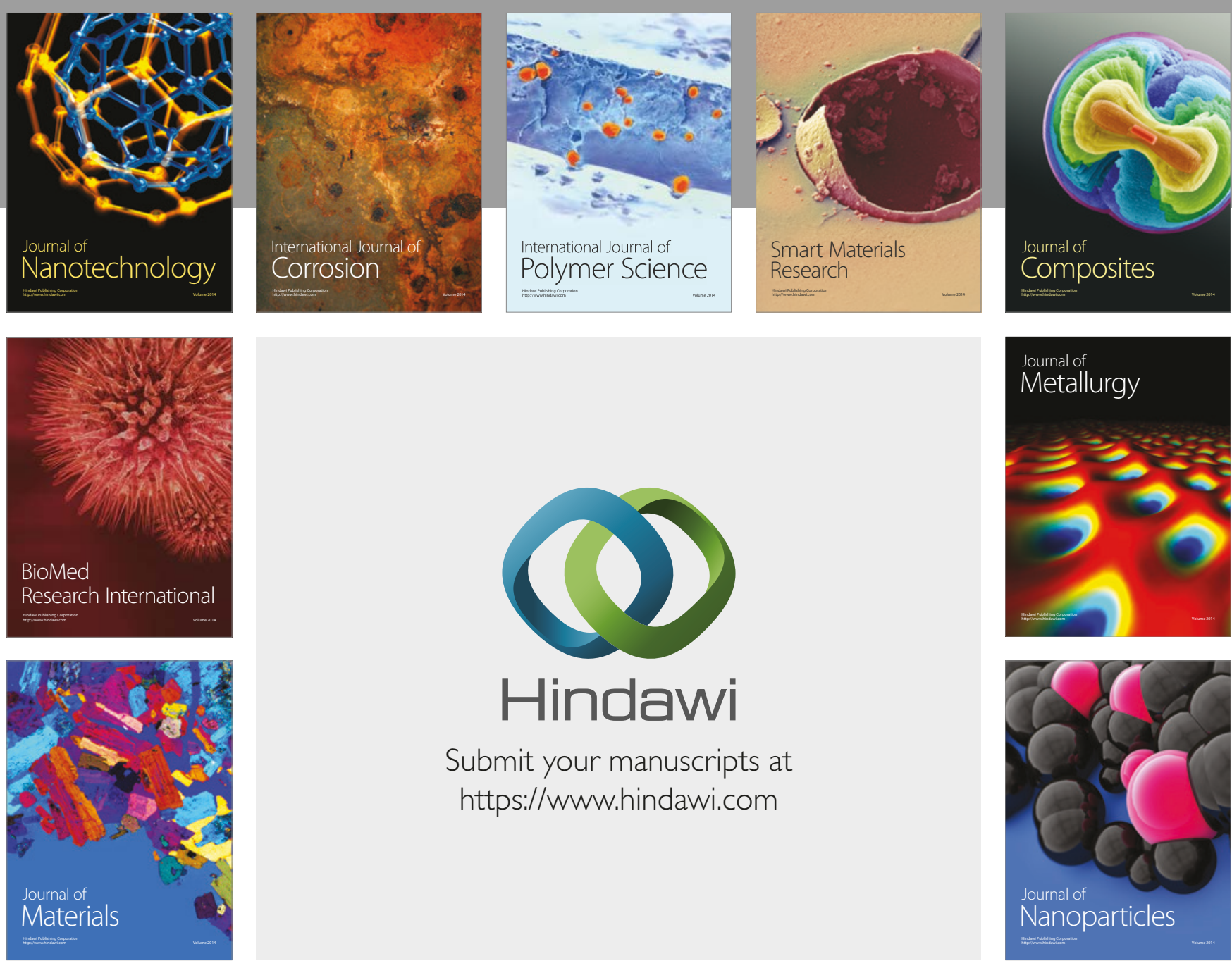

\section{Hindawi}

Submit your manuscripts at

https://www.hindawi.com
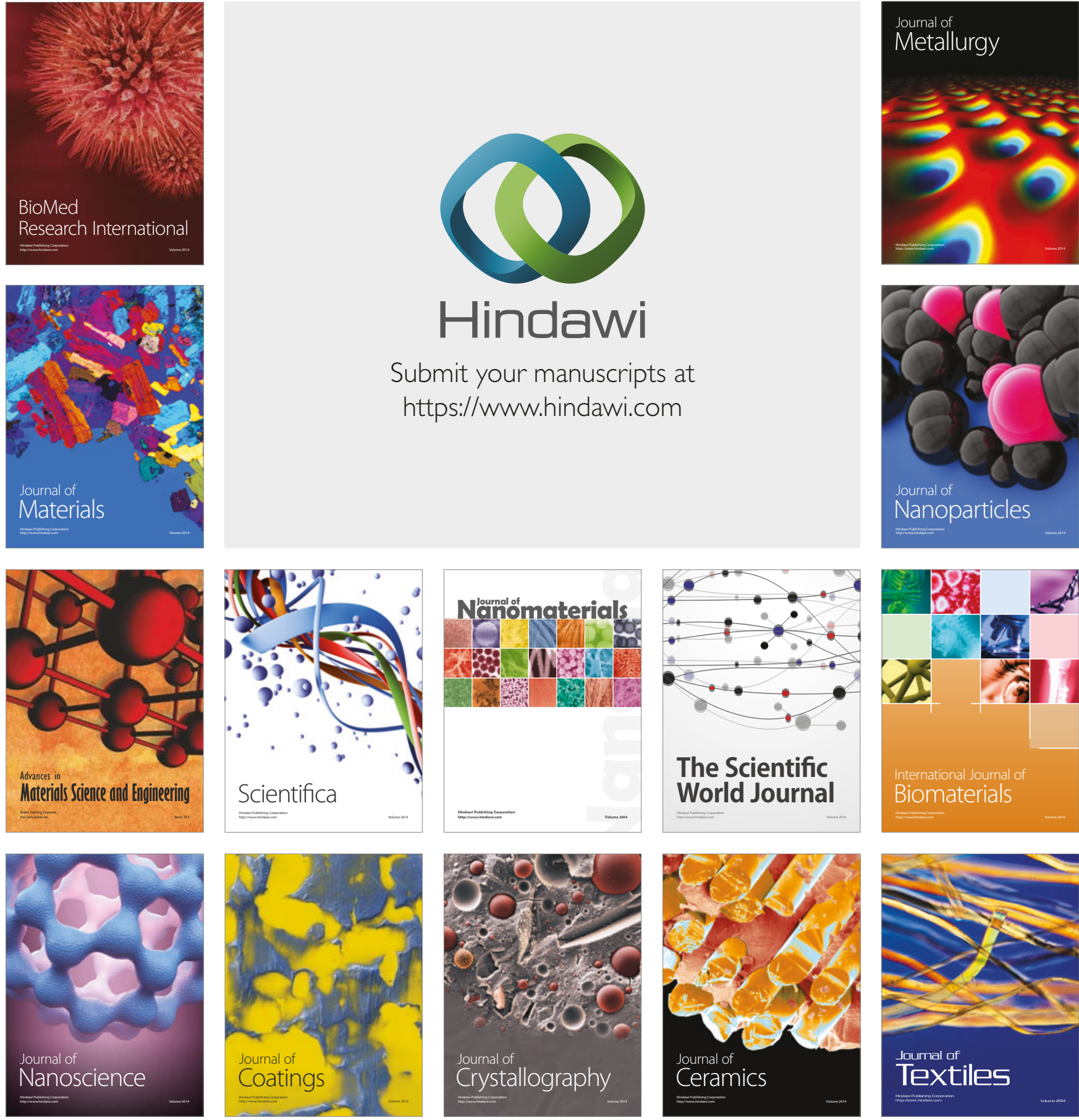

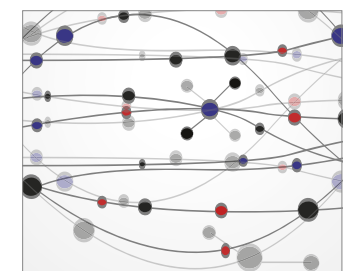

The Scientific World Journal
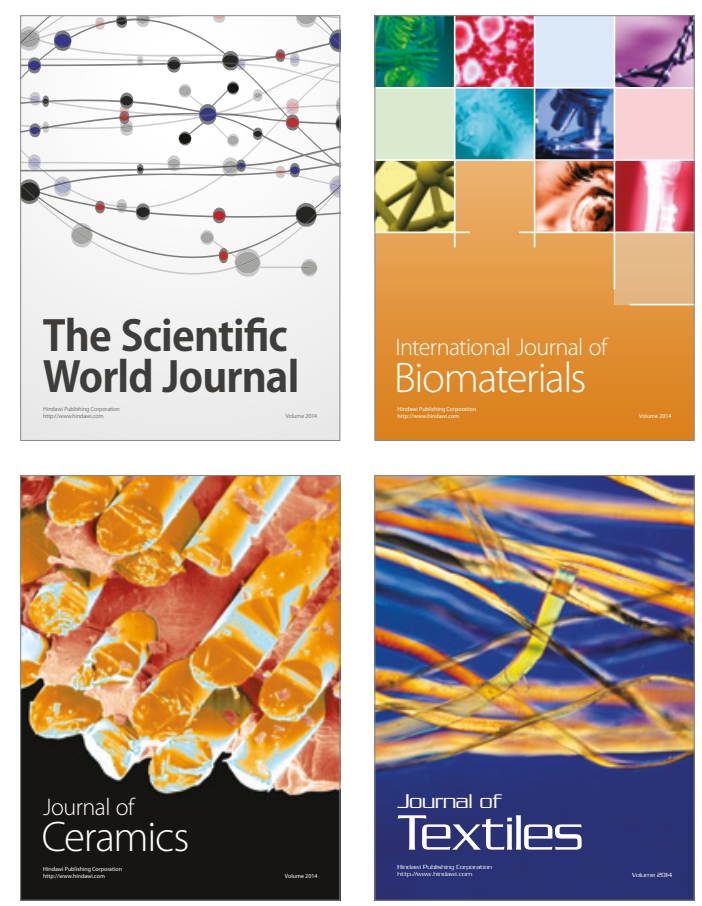